\title{
Technical Program Description Systems Integration for Manufacturing (SIMA)
}

Howard M. Bloom

U.S. DEPARTMENT OF COMMERCE Technology Administration National Institute of Standards and Technology

Gaithersburg, MD 20899

\author{
(oward M. Bloom
}





\section{Technical Program Description Systems Integration for Manufacturing (SIMA)}

Howard M. Bloom

U.S. DEPARTMENT OF COMMERCE Technology Administration National Institute of Standards and Technology Gaithersburg, MD 20899

July 1994

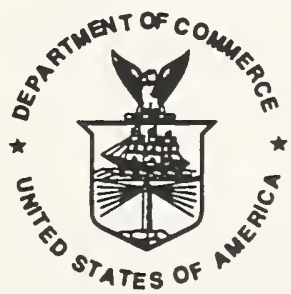

U.S. DEPARTMENT OF COMMERCE Ronald H. Brown, Secretary

TECHNOLOGY ADMINISTRATION Mary L Good, Under Secretary for Technology NATIONAL INSTITUTE OF STANDARDS AND TECHNOLOGY

Aratl Prabhakar, DIrector 


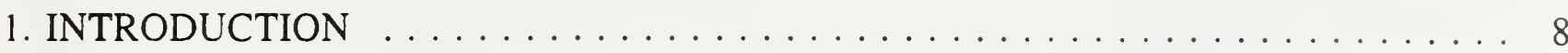

1.1 Vision for Manufacturing within the NII $\ldots \ldots \ldots \ldots \ldots \ldots$

1.2 Industry Needs for Systems Integration . . . . . . . . . . . . . 10

2. BACKGROUND ON THE NATIONAL INFORMATION INFRASTRUCTURE

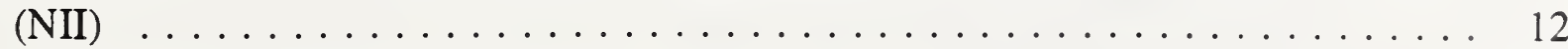

2.1 National Challenges . . . . . . . . . . . . . . . . . 13

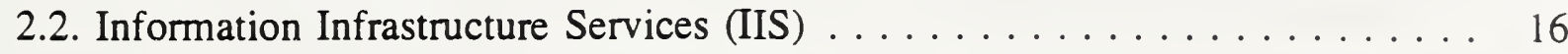

2.3 System Development and Support Environments (SDSE) . . . . . . . . . 17

2.4 Intelligent Interfaces (II) . . . . . . . . . . . . . . . . . . . . . . 19

2.5 Advanced Manufacturing Technology Initiative . . . . . . . . . . . 20

3. DESCRIPTION OF NIST PROGRAM $\ldots \ldots \ldots \ldots \ldots \ldots \ldots \ldots \ldots \ldots \ldots$

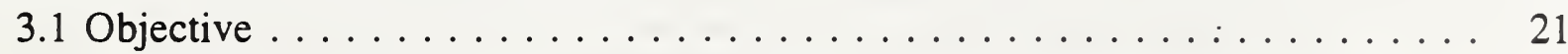

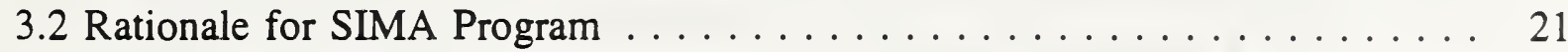

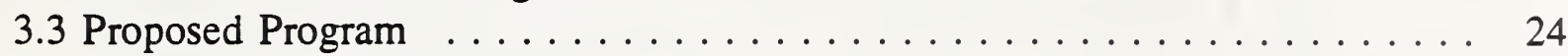

3.4 Five Year Outlook ......................... 25

4. PRODUCT DATA STANDARDS AS KEY . . . . . . . . . . . . . . . 27

4.1 Importance of Product Data Exchange Standards . . . . . . . . . . . . 27

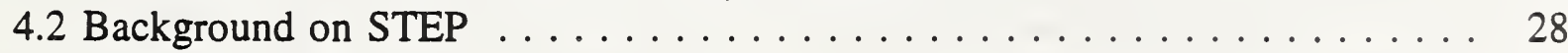

4.3 STEP Technology . . . . . . . . . . . . . . . . . . 30

5. PROJECT DESCRIPTIONS . . . . . . . . . . . . . . . . . . . 30

5.1 Manufacturing Systems Environment ............... 31

5.1 .1 Objective .......................... 31

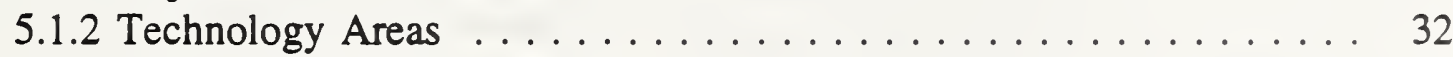

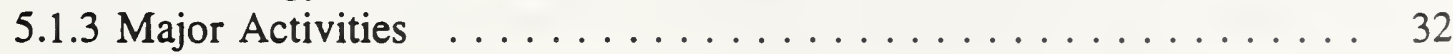

5.1 .4 Expectations ..................... 32

5.1.5 Projects to be Initiated for FY $1994 \ldots \ldots \ldots \ldots \ldots \ldots \ldots$

5.1.6 Supplemental Projects Proposed for FY $1995 \ldots \ldots \ldots \ldots \ldots$

5.2 Technology Transfer Environment . . . . . . . . . . . . . . . 40

5.2 .1 Objective . .................... 40

5.2.2 Technology Transfer Strategy . . . . . . . . . . . . 40

5.2 .3 Activities . . . . . . . . . . . . . . . . . . . . 41

5.2 .4 Expectations . . . . . . . . . . . . . . . 41

5.2.5 Projects to be Initiated for FY $1994 \ldots \ldots \ldots \ldots \ldots \ldots . \ldots 42$

5.2.6 Supplemental Projects Proposed for FY $1995 \ldots \ldots \ldots \ldots . \ldots 42$

5.3 Standards Development Environment . . . . . . . . . . . . . . 43

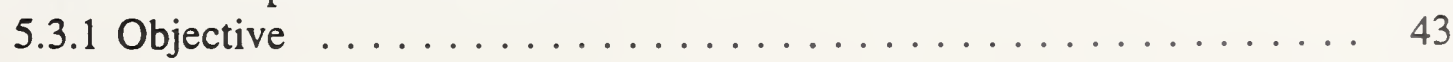




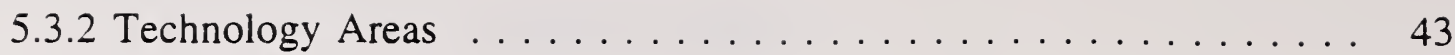

5.3 .3 Activities ....................... 44

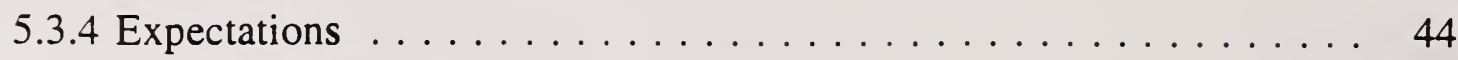

5.3.5 Projects to be Initiated for FY $1994 \ldots \ldots \ldots \ldots \ldots \ldots \ldots$. . . . . 45

5.3.6 Supplemental Projects Proposed for FY $1995 \ldots \ldots \ldots \ldots \ldots 47$

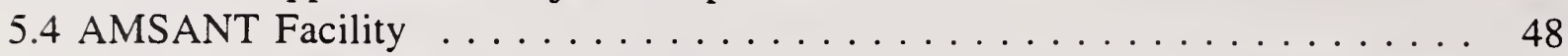

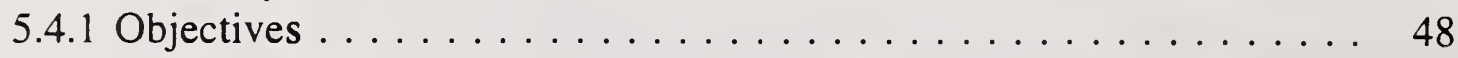

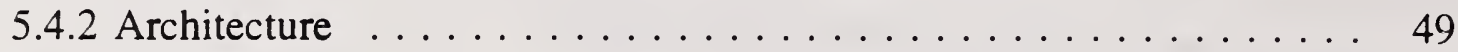

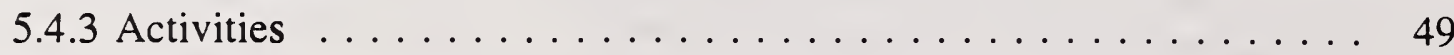

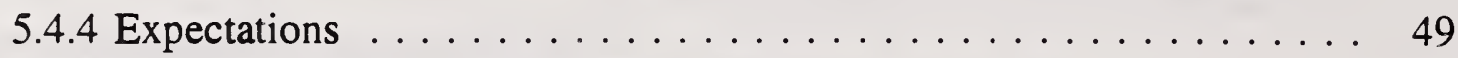

5.4 .5 Projects to be Initiated for FY $1994 \ldots \ldots \ldots \ldots \ldots \ldots \ldots \ldots$

5.4 .6 Projects Proposed for FY $1995 \ldots \ldots \ldots \ldots \ldots \ldots \ldots \ldots \ldots$

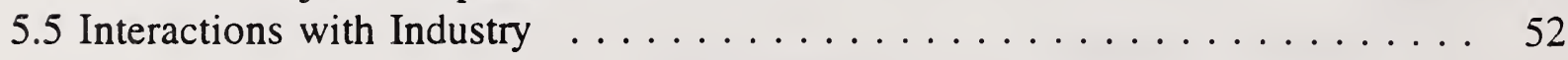

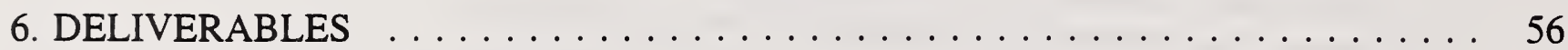

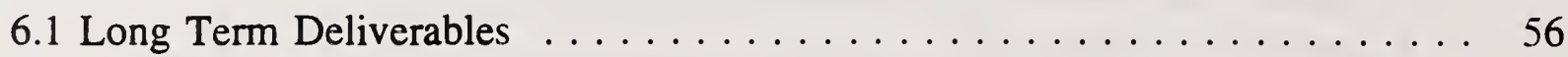

6.2. Generic Deliverables ........................ 57

6.3 Five Year Deliverables for Projects . . . . . . . . . . . . . . . . . 59

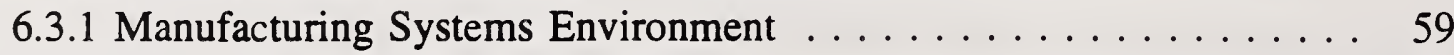

6.3.2 Technology Transfer Environment . . . . . . . . . . . . . 67

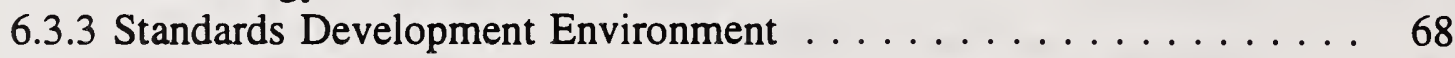

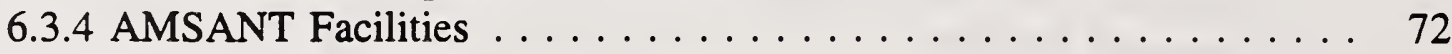

6.4 Deliverables for FY95 Incremental Projects . . . . . . . . . . . . 74

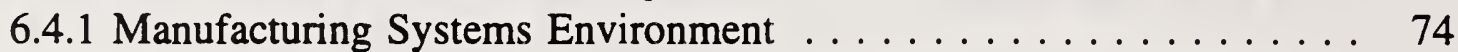

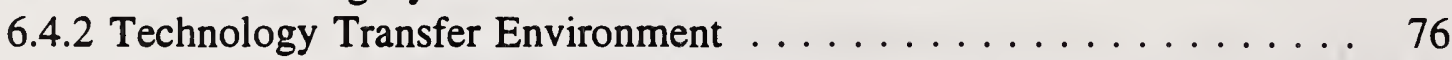

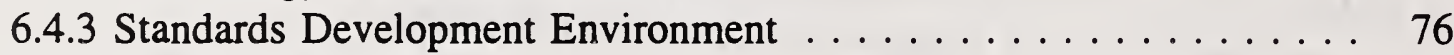

6.4 .4 AMSANT Facilities $\ldots \ldots \ldots \ldots \ldots \ldots \ldots \ldots \ldots \ldots \ldots$

7. SUPPORTING PROGRAMS . . . . . . . . . . . . . . . . . . . . . . . . 77

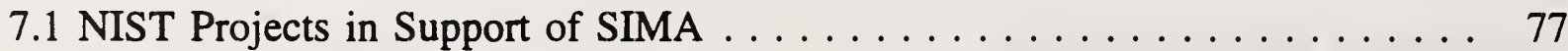

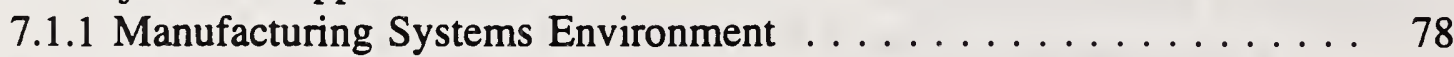

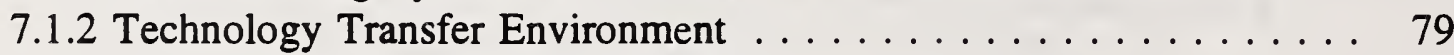

7.1.3 Standards Development Environment . . . . . . . . . . . 80

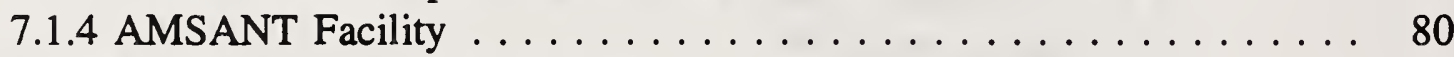

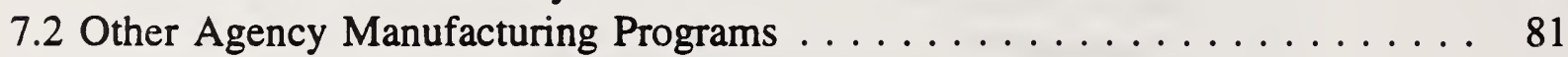

8. PROGRAM MANAGEMENT $\ldots \ldots \ldots \ldots \ldots \ldots \ldots \ldots \ldots \ldots \ldots$

9. PROGRAM STATUS $\ldots \ldots \ldots \ldots \ldots \ldots \ldots \ldots \ldots \ldots \ldots \ldots \ldots$

10. ACKNOWLEDGEMENTS $\ldots \ldots \ldots \ldots \ldots \ldots \ldots \ldots \ldots \ldots \ldots$

11. REFERENCES $\ldots \ldots \ldots \ldots \ldots \ldots \ldots \ldots \ldots \ldots \ldots \ldots \ldots \ldots$ 


\section{ABSTRACT}

NIST has established an Advanced Manufacturing System and Networking Testbed (AMSANT) which supports R\&D in high performance manufacturing systems and testing high-performance computer and networking hardware and software in a manufacturing environment. The testbed will serve as a demonstration site for use by industrial technology suppliers and users, and assist industry in the development and implementation of voluntary standards. A standards-based data exchange effort for computer integrated manufacturing will focus on improving data exchange among computer aided design, process and manufacturing activities. This new program, Systems Integration for Manufacturing Applications, is part of the government's High Performance Computing and Communications Initiative. Applications may include enterprise integration for manufacturing applications, integrated product/process design, simulation and agile manufacturing. Prototype systems and interface specifications will be communicated to appropriate standards organizations. Results will be made available to US industry through workshops, training materials, electronic data repositories and pre-commercial prototype systems that can be installed by potential vendors for test and evaluation. NIST will distribute Standards

Reference Data, technical information, and digital product data designs via digital library technologies. This report describes the objectives of the program and highlights the projects to be initiated in FY 94 and those proposed for FY 95

\section{EXECUTIVE SUMMARY}

BACKGROUND The National Institute of Standards and Technology (NIST)'s program is part of the multi-agency High Performance Computing and Communications (HPCC) initiative as described in the High Performance Computing Act of 1991 and the Senate bill S.4, "The National Competitiveness Act of 1993." A major portion of the NIST HPCC program described in this report for FY 1994 and beyond is included under the Information Infrastructure Technology Applications (IITA) category of the HPCC initiative. The objectives of the program are: (1) to accelerate the development and deployment of HPCC technologies required for the National Information Infrastructure (NII) and (2) to apply and test these technologies in a manufacturing environment. Ultimately, these technologies will radically transform America's manufacturing environment, allowing individual companies to interact electronically as part of a "virtual enterprise" to produce world-class products for the 21 st century.

The program will focus on technologies and standards that will improve the systems integration function in manufacturing. NIST will perform appropriate activities in the areas of flexible computer-integrated manufacturing (FCIM) with emphasis on both product data exchange (for manufacturing) and electronic data interchange (for electronic commerce) standards that are part of the overall vision for 21 st century manufacturing. The infrastructure technologies being developed will serve as an enabler for such manufacturing paradigms as Agile Manufacturing, Concurrent Engineering, and the Virtual Enterprise. The centerpiece of these activities will be 
a model facility at NIST, the Advanced Manufacturing Systems and Networking Testbed (AMSANT). Researchers nationwide will use the AMSANT facility to research and develop methods for applying HPCC technology to manufacturing. Besides the technology development, important functions of the program include improving the process for developing the key manufacturing interface standards and providing a technology transfer mechanism for getting the program results to industry.

DISCUSSION OF NATIONAL INFORMATION INFRASTRUCTURE (NII) The National Information Infrastructure (NII) is designed to promote a seamless web of communications networks, computers, databases, and consumer electronics that will put vast amountș of information at users' fingertips. Development of the NII can help unleash an information revolution that will change forever the way people live, work, and interact with each other. The NII is the platform of information technology resources upon which industry, government, and academia can integrate their information functions.

The HPCC/IITA initiative supports the key areas of research and development and systems integration to demonstrate prototype solutions to National Challenges (e.g., Advanced Manufacturing) starting from the advanced technology level moving through higher level of user capabilities to the ultimate user level in National Challenge projects. The IITA consists of four elements: (1) National Challenges are fundamental applications that have broad and direct impact on the Nation's well-being and competitiveness, (2) Information Infrastructure Services provide the underlying network-capable building blocks upon which the National Challenges can be constructed, (3) Intelligent Interfaces will bridge the gaps between users and the future NII, and (4) System Development and Support Environments will provide the network-based software development tools and environments needed to build the advanced user interfaces and the information-intensive National Challenges themselves.

The NIST program is concerned with one specific National Challenge, Advanced Manufacturing:

Supports work in advanced manufacturing technologies through the use of HPCC capabilities in design, production, planning \& quality control, marketing \& user services. A key element is the development of the infrastructure necessary to make the process and product information accessible over the information highway to both enterprises and customers. Research areas include concurrent engineering, protocols for electronic exchange of product data, electronic commerce for manufacturing, virtual design technologies, etc.

Implementation of the NII concept for manufacturing will allow such capabilities as: (1) customers to "custom design" products, (2) companies to form alliances needed to produce new products (i.e., Agile Manufacturing), (3) small to medium size companies to interact with large companies for bidding on products (i.e., the Virtual Enterprise), (4) software system brokers to "rent" sophisticated manufacturing systems tools, and (5) rapid access to manufacturing knowledge by the product designers that will enable enterprises to use concurrent engineering practices. 
DESCRIPTION OF NIST PROGRAM The primary objective of the NIST's Systems Integration for Manufacturing Program (SIMA) program is to develop technologies for applications of HPCC, with the first application designated as manufacturing. The effective utilization of these technologies can dramatically improve product quality and performance and reduce costs and time-to-market, thus greatly increasing American industry's ability to compete effectively in domestic and international markets.

Technology innovation is not the only requirement to meet the vision for making the NII a reality for advanced manufacturing. Standards are needed to facilitate economic interactions that require a high level of cooperation and coordination. Standards also help to determine the efficiency and effectiveness of the economy as well as the cost, quality and availability of products.

Standards have become more important due to our growing reliance on technology. The growing pace of technological change also drives the need for standards. The faster the advance of technologies, the greater the risk in $\mathrm{R} \& \mathrm{D}$ and product development. In fact, it is now necessary to develop standards even before the actual products are made available to the customer. However, industry has been reluctant to adopt standards because of the slowness in which the standards community can react by approving standards through the consensus process. New approaches to the implementation of standards need to be developed that significantly accelerate the standards approval process. The future will involve the concurrent development of new technology and corresponding standards that allow vendors to build products to meet the technology needs. The attainment of this new paradiigm will be a major focus of the SIMA program.

PRODUCT DATA STANDARDS AS KEY The program is built upon the premise that interface standards are necessary to make systems integration an effective tool for advanced manufacturing systems. The Standard for Exchange of Product Model Data (STEP) is considered to be the key standard for the integration activities, although there are many other interface standards that will be part of the program.

The STEP effort is expected to accelerate the evolution of concurrent engineering, support Electronic Data Interchange (EDI) electronic commerce and enable business partners to share sophisticated digital product data as easily as paper drawings are shared today.

Application Protocols (which convert end-user requirements into specifications) will be implemented across a wide range of products such as mechanical, electronics \& electrical, apparel, distribution systems, and structures. The long term goal is to develop sets of application protocols that will be complete for a given type of product in a given application such as design or manufacturing. These application protocols allow for the testing of the proposed standards and to further understanding and improvements in the standards and methods. It is through the development of the applications and relevant application protocols by the industry-led standards community that technology can be implemented and then transferred to industry as standardconformant products. 
PROJECT DESCRIPTIONS The SIMA projects fall within the following four program elements: (1) Manufacturing Systems Environment, (2) Technology Transfer Environment, (3) Standards Development Environment, and (4) AMSANT Facility. Many of the projects have been initiated in FY94. In general, projects started in FY94 will be expanded and several additional new projects initiated in FY95 (assuming the planned budget initiative submitted by the President is approved by Congress).

Manufacturing Systems Environment - The major thrust is in the development of the technologies and standards for integrating manufacturing systems that support manufacturing domains such as mechanical products, apparel, electronics, construction, and chemical processing.

There are ten projects initiated for FY94: (1) - (4) Control Systems A rchitecture, Integration Specifications, Design Applications, and Planning Applications - develop the integration framework for mechanical parts production, (5) Process Plant Engineering and Construction develop the integration framework for the process plant industry in the engineering phase, (6) Interoperability of Chemical Measurements and Data Systems - develop the communications integration protocol for the chemical instrumentation industry, (7) Electronic Commerce for the Electronics Industry - develop prototype demonstrations showing how on-line journals can be used as an intelligent index to technical component information and can serve as an entry point for on-line commerce, (8) Virtual Design Technology - develop specific HPCC enhancement for manufacturing of mechanical parts, (9) Packaging and Interconnect Materials Supporting HPCC Infra-Technology - develop improved materials data needed for manufacturing electronic components, and (10) Collaborative Environment Platform - provide an improved software platform in which to develop the systems integration technologies.

There are nine new projects proposed for FY95 that will address different manufacturing domains and computing applications that encompass the product life-cycle: (1) Manufacturing Systems Engineering Tools - provide an integrated framework, operating environment, common databases, and interface standards for a wide variety of emerging software tools and techniques for designing manufacturing processes, equipment, and enterprises, (2) Advanced Apparel $M$ anufacturing Systems - develop apparel design and production software within the AMSANT in order to implement integration solutions, (3) Electronic Commerce for Mechanical Parts Manufacturing - harmonize the electronic commerce applications and the manufacturing applications along with the associated information, (4) Process Plant Conceptual Design - develop systems integration technologies for the process plant industry in the design phase, (5) Building Systems Design and Operation - develop an integrated model for the performance of buildings and their systems, (6) A pplication Protocols for Electronic Products - develop systems integration technologies for the electronics Industry, (7) - (8) Open-Systems Manufacturing of Enabling $M$ aterials and Integration of Materials Performance Data into Computer-A ided Design - develop improved use of materials information in manufacturing systems, and (9) Integration of Reference Data Systems into Information Networks - develop an integrated view of the scientific and engineering standard reference data.

Technology Transfer Environment - The objectives are: (1) develop technology transfer 
infrastructure that can be used to exchange manufacturing information among manufacturing organizations using HPCC technology, (2) develop prototype information services in collaboration with industry partners that could become commercialized products, (3) develop services related to document searches and retrieval of government and other research reports, and (4) establish communication channels for network of researchers and implementors of manufacturing technologies.

There is only one project, Manufacturing Information Technology Transfer, to be initiated in FY 1994 which will define and implement a set of software tools and services for disseminating SIMA and HPCC technology information, and related research results and information exchange standards between NIST and industry collaborators. Three new projects are proposed for FY95 that are concerned with the same issues as the FY94 project: Technology Transfer for the A pparel Products Industry, Technology Transfer of HPCC Information into the Electronics Industry, and Technology Transfer for the Construction Industry.

Standards Development Environment - The objectives are:(1) assist industry in implementing voluntary consensus standards relevant to computer integrated manufacturing (CIM), (2) facilitate industry efforts to test new applications of advanced manufacturing systems and networks, (3) facilitate efforts to develop and test new data exchange standards utilizing HPCC technology, and (4) accelerate industry deployment of consensus standards. There is a general theme of providing effective support environments for the development of standards as well as facilitating harmonization across the broad spectrum of standards that compose the range of information and supporting technology required for enterprise integration. Several projects will be involved in coordinating the support mechanisms and information across the various industries.

There are six projects initiated in FY 1994: (1) - (3) Conformance Testing for STEP, Application Protocol Development Environment, and STEP for the Process Plant Industries - develop methodologies and tools for interface standards required for systems integration, (4) Application Interface Development Environment - develop tools that will enable information to be exchanged across the data highway, and (5) - (6) High-Speed Electronic and Optical Components, and Video Processing - develop improved measurement capability for selected HPCC electronic components.

There are four new projects proposed for FY95: (1) Harmonization of Standards - address problems involved in the effective integration of manufacturing information standards, (2) - (3) Infrastructure for Interoperable Electrical \& Electronic Product Data Exchange Standards, and Concurrent Engineering of Information Exchange Standards - develop standards for the electronics industry, and (4) Software Production and Integration Environment - develop standards for the software industry.

AMSANT Facility - The objectives are: (1) serve as a demonstration site that industrial technology suppliers and users can utilize, (2) serve as the interface to a network of technology development testbeds within the United States, and (3) serve as the interface to one or more information dissemination contacts. 
There are three projects for FY 1994: AMSANT for Mechanical Parts Production, Operator Interfaces for AMSANT, and AMSANT for Process Plant Industries. There is only one new project proposed for FY95: AMSANT for A pparel Production.

INTERACTIONS WITH INDUSTRY The SIMA program will only be successful if there is a strong interaction with industry to produce the technologies and standards required for systems integration. Emphasis will be placed on developing Cooperative Research and Development Agreements (CRDAs) with industry and especially consortia and Memoranda of Understanding with the appropriate standards bodies.

DELIVERABLES There are plans for each of the FY94 SIMA projects that include detailed set of five year deliverables. In addition there are proposed plans for new FY95 projects (or expanded FY94 projects) that include a description of new deliverables. The following are long term deliverables for the Advanced Manufacturing application subelement within the National Challenges element of the IITA program. The SIMA program uses the same set of deliverables (assumes that new initiatives are begun each year up to FY 1998 and brings in expected collaboration with other agencies):

\section{Install Applications: FY 1994 - FY 1998}

M1. Establish and augment advanced manufacturing systems and networking testbeds (AMSANT) throughout some government agencies that perform manufacturing research as well as with agency contractors who are building systems to support agency functional requirements. This will demonstrate to industry how systems integration can be effectively utilized to allow for such strategies as Agile Manufacturing and Concurrent Engineering to be applied across enterprise boundaries. Select sets of manufacturing applications based on current industry needs and assist with the installation of state-of-the-art systems. Establish links to other testbeds within industry and academia to help evaluate, measure, and disseminate effects of new technology in order to demonstrate "virtual" AMSANTs. Provide on-line capability for industry to access manufacturing technology information using such systems as Xmosiac and World Wide Web.

\section{Develop Interface Specifications: FY 1994 - FY 1997}

M2. Develop interface specifications for the set of manufacturing applications chosen in M1 that will be used to implement an integrated manufacturing environment. Publish these specifications as proposed standards in such areas as product data exchange and interoperation of heterogeneous databases and applications. Choose applications that extend across the manufacturing life-cycle for a given industry (e.g., mechanical part production) as well as across industries (i.e., electronics, process plant design). Publish frameworks for the integration of the standards required for the harmonization of the applications involved in the implementation of both the manufacturing and information technologies.

\section{Extend Applications: FY 1995 - FY 1998}

M3. Based on interface specifications from M2 and applications installed from M1, build and support the development of application interfaces (APIs) so that the applications can easily adopt these extensions. Demonstrate the integration of applications across local and NII networks that 
link multiple AMSANTs. Begin exploring the concept of "brokering" tools from different vendors to built integrated tools for concurrent engineering tasks/time-specific use (e.g., integrate different types of simulation and analysis tools that are needed for requirements during design, manufacturing, distribution, maintenance and recycling).

\section{Develop Conformance Testing: FY 1996 - FY 1998}

M4. Develop, evaluate, and publish conformance testing methods and services to assess vendor conformance to the proposed specifications (and emerging standards) developed in M2. Support the participation of small businesses and academics in the standardization process. Demonstrate an advanced selection of prototype vendor products that conform to the specifications. Implement mechanisms and systems that accelerate the development of the critical standards needed for manufacturing integration.

\section{Implement Database Environment: FY 1997 - FY 1998}

M5. Implement an interoperating, heterogeneous manufacturing database environment to support the applications implemented under M3. Develop an integrated database for product and process information required for sets of manufacturing applications. Publish specifications for the industry/application database. Demonstrate that this environment can communicate among and co-exist at multiple sites.

\section{Demonstrate Virtual Enterprise: FY 1998 - FY 1999}

M6. Demonstrate the fully integrated set of applications that access both product and process information through the manufacturing database, using both large and small industrial sites. Specifically provide demonstrations illustrating (1) the ability for customers to "custom design" products, (2) agile manufacturing among enterprises, (3) supplier-contractor interactions such as bidding systems, (4) concurrent engineering for product development, and (5) enterprise integration including both advanced manufacturing and electronic commerce applications.

INTERACTION WITH OTHER AGENCY PROGRAMS The HPCC Program places heavy emphasis on collaboration and the interdependencies between the efforts of individual agencies. This will clearly be a key issue in the manufacturing applications because there are many related efforts underway or planned, particularly within ARPA (Manufacturing Automation Design Engineering, Agile Manufacturing, Persistent Object Bases), other DoD elements (CALS/PDE, FCIM), and DoE (Advanced Manufacturing Technology Network/Sandia Labs and Technologies Enabling Agile Manufacturing/Y12).

It will be part of NIST's strategy to coordinate and build upon these other efforts. To this end, the SIMA program will identify relevant on-going and planned efforts, and, wherever possible, attempt to forge collaborative efforts that leverage the investments and expertise of the various parties involved.

In an effort to work with other government agencies, standards bodies, industry, and universities, NIST will use the following strategy: (1) perform research and development into Product Data 
Exchange (PDE) and FCIM technologies in collaboration with other institutions, (2) perform research and development into the technologies required to develop the standards, (3) implement the prototype advanced manufacturing systems that conform to the standards, (4) take an active role in developing required standards, (5) take a lead role in developing the testing and evaluation methods to ensure that quality standards are developed and implemented by vendors, and (6) work with industrial facilities in implementing the FCIM and PDE standards.

\section{INTRODUCTION}

The National Institute of Standards and Technology (NIST)'s technical program described in this report is part of the multi-agency High Performance Computing and Communications (HPCC) program as described in the High Performance Computing Act of 1991 and the Information Infrastructure and Technology Act of 1992 introduced by former Senator Gore. The latter was incorporated in the Senate bill S.4, "The National Competitiveness Act of 1993." This program will enable NIST to expand and integrate its programs in advanced manufacturing applications research \& development, computer performance measurement, communications protocols, advanced software, and the development of the National Research and Education Network (NREN). NIST's program for FY 1994 and beyond is included under the Information Infrastructure Technology Applications (IITA) category of the HPCC initiative.

Efficient access to information is becoming critical for all parts of the American economy. Banks, insurance companies, manufacturing concerns, and many business operations depend on high-speed communications links. These systems must be reliable, easy to use, and inexpensive. The objectives of the NIST program are: (1) to accelerate the development and deployment of high performance computing and networking technologies required for the national information infrastructure and (2) to apply and test these technologies in a manufacturing environment. Building on its leadership in research, standards development, measurement technology and technology transfer in both information technology and manufacturing, NIST will utilize a test facility to enable the accelerated application of information technologies to manufacturing to accomplish this objective. Success of this deployment will be measured by the commercialization and market penetration of such technologies.

HPCC technology will radically transform America's manufacturing environment, allowing individual companies to interact electronically as part of a "virtual enterprise" to produce worldclass products. NIST has envisioned a framework (shown in Figure 1) that implements this view of 21 st century manufacturing. Independent enterprises operating as suppliers, system integrators, merchants and customers are integrated by an information network into an effective system. Within each of these enterprises the various product-related functions and product life-cycle stages are integrated through the sharing of product and process data, although each stage maintains its own view of the product. Based upon standards, the inter- and intra- enterprise framework enables the integration of the product life-cycle processes such as design, manufacturing, marketing, maintenance, and improvement. This is commonly referred to as the practice of multi-enterprise concurrent engineering through which the characteristics of world class products are achieved [CARV91]. These characteristics are short time-to-market, low cost, 
high quality, and high performance.

NIST will perform appropriate activities in the areas of flexible computer-integrated manufacturing (FCIM) with emphasis on both product data exchange (for manufacturing) and electronic data interchange (for electronic commerce) standards that are part of the overall vision for 21 st century manufacturing. The program will focus on technologies and standards that will improve the systems integration function in manufacturing. The infrastructure technologies being developed will serve as an enabler for such manufacturing paradigms as Agile Manufacturing, Concurrent Engineering, and Virtual Enterprises. The centerpiece of these activities will be a model facility at NIST, the Advanced Manufacturing Systems and Networking Testbed (AMSANT). Researchers nationwide will use the AMSANT facility to research and develop methods for applying HPCC technology to manufacturing.

Flexible and rapid access to information about suppliers, product requirements, and product failures is a critical issue in making U.S. manufacturing competitive. The IITA initiative is closely tied to the broader National Information Infrastructure (NII) that is a joint industry/government program. Improved productivity in manufacturing depends on our efforts to employ the NII in pursuit of our national goals in manufacturing.

\subsection{Vision for Manufacturing within the NII}

Manufacturing is a mainstay of our economy and a source of high-paying and productive jobs, but the distributed and varied nature of the U.S. manufacturing base is both an asset and a liability. As a benefit it provides rapid innovation, flexibility in organization, and lower costs through competition. However, without an effective NII to bridge the communications gap between companies, the benefits of diversity can be lost, especially for small companies.

Implementation of the NII concept will allow:

o Customers to "custom design" products such as automobiles and clothing and transmit their requirements to industries with the capability of manufacturing these products ;

- Companies to form alliances needed to produce new products using new Agile Manufacturing concepts;

o Small to medium size companies to advertize their manufacturing capabilities over the network and thus automatically bid on projects required by the prime companies in areas such as automotive and aerospace (thus promoting the concept of the virtual enterprise);

o "Software system brokers" to connect users who have a need for temporary access to sophisticated manufacturing systems tools that would normally be too expensive to use;

o Rapid access to manufacturing knowledge by the product designers which will enable enterprises to use concurrent engineering practices; 
o Financial settlements to be made with minimal paper work by linking the transactions to electronic banking services, reducing the outstanding balances and financial risks for small businesses.

o Records of product failures and complaints to be rapidly acted upon, reducing wastage in unusable inventories, rework, and permitting rapid product improvement;

o New business opportunities to arise in processing, analyzing, and disseminating manufacturing information.

The NII potential for making available the best manufacturing application tools, knowledge bases, product information, and training materials regardless of location would importantly support this objective, provided access to these capabilities is universal. This means that all manufacturing enterprises, both large and small, will need to be connected to the networks that constitute the NII through easy-to-use access programs that are capable of running on whatever types of computers are available. Given the number and diversity of the businesses involved and the expense of providing the physical connectivity through either land line or direct broadcast, connectivity and universal access represent one of the major problems to be overcome in the near-term in order to avail ourselves of the promise for improving U.S. manufacturing practices inherent in the NII.

The NII can make available the infrastructure for rapid dissemination for requirements and specifications for parts and sub-assemblies, letting the most qualified and competitive suppliers respond. The high cost of advising suppliers of new capabilities will be reduced, leading to more rapid adoption of innovation in materials, parts, and manufacturing processes. Interaction between suppliers and purchasers can lead to adaptations in products that reduce cost and improve manufacturability.

Easy-to-use and access services within the NII are essential, and must provide interfaces to whatever types of computers are in use now and are becoming available. Standard interfaces and infra-structure services will be needed, but many of those will be identical to those needed for other NII national challenges. Reliable connectivity and universal access, at a low initial investment must be provided in the near-term in order to avail ourselves of the promise for improving manufacturing effectiveness through the NII.

\subsection{Industry Needs for Systems Integration}

A workshop entitled "System Integration Needs of U.S. Manufacturers" was held at NIST [SINM93] to identify the key systems integration needs of U.S. manufacturers in the areas of technology development, standards development, and technology transfer. The workshop attendees were given a strategic plan [BLO093] as input that identified the principal objectives of the HPCC/IITA initiative for manufacturing and the complementary Advanced Manufacturing Technology Initiative (see Section 2.5). These needs were defined in terms of the support required by NIST in the realization of the vision of manufacturing within the NII. The list of 
identified needs has been used to provide clearer links between the NIST program and the needs of U.S. industry and to help identify mechanisms by which NIST can respond to industry needs.

There were four broad initiatives defined for NIST in support of U.S. manufacturing:

o Develop and demonstrate a virtual enterprise testbed;

- Expand scope of metrics and lessons learned to extend a system of integration technologies to U.S. manufacturing;

- Establish and test metrics for learning and retention of integrated manufacturing concepts, technologies, and practices;

o Establish collaborative programs among universities, federal laboratories, and businesses focusing on mutual beneficial technologies.

In the area of technology development, the following key thrusts were identified:

o Establish a data model for systems integration;

o Determine attributes of a complete and consistent model for an industrial process;

o Develop neutral test and validation technology;

o Establish testing methodologies for interoperability.

In the area of technology transfer, the following key thrusts were identified:

o Study transfer mechanisms, develop pilot programs for these mechanisms, and perform demonstrations that illustrate the transfer of technology;

o Establish computer support systems for an electronic network, on-line databases, and user-friendly search techniques;

o Provide other mechanisms through symposia on success stories, newsletters, and testbeds;

o Serve as catalyst for commercialization of technology.

In the area of standards, the following key thrusts were identified:

o Establish a new perspective on developing standards that includes (1) investigating best practices, (2) reengineering critical standards development processes, (3) working with industry leaders, (4) championing good management practices, (5) assuming responsibility for assisting standards groups, and (6) providing more training, testing, and evaluation.

o Establish metrics to measure progress and quality by working with industry to develop a mechanism for tracking standards development, and adopting a product implementation perspective on standards development; 
o Establish better communication among U.S. standards-making groups by developing and maintaining repositories of information on U.S. and international standards development efforts, brokering relationships among consortia, and maintaining computer-based communications for standards groups;

o Provide an effective funding mechanism for standards development by establishing a standards development program modeled on the Advanced Technology Program (ATP), focusing AMSANT on standards, developing demonstration and implementation projects, increasing NIST participation in standards development groups and consortia, and funding critical processes not funded directly by industry.

Section 2 provides a background on the NII and how it relates to the HPCC Initiative. Section 3 describes the program that is being implemented at NIST in response to the industry needs for systems integration in manufacturing. Section 4 describes the role of the Standard for the Exchange of Product Model Data (STEP) as the key standard in the NII/HPCC manufacturing implementation. Section 5 describes the various projects that have been defined for the NIST program. Section 6 describes the key deliverables for the program. Section 7 describes the supporting projects within NIST that are part of other programs and the programs being established by the other government agencies within the HPCC/IITA initiative. Section 8 discusses the management structure implemented for this program within NIST.

\section{BACKGROUND ON THE NATIONAL INFORMATION INFRASTRUCTURE (NII)}

The National Information Infrastructure (NII) is designed "to promote a seamless web of communications networks, computers, databases, and consumer electronics that will put vast amounts of information at users' fingertips [NII93]. Development of the NII can help unleash an information revolution that will change forever the way people live, work, and interact with each other." The NII is the platform of information technology resources upon which industry, government, and academia can integrate their information functions.

There are nine guiding principles and objectives:

(1) Promote private sector investment;

(2) Extend the universal service concept to ensure that information resources are available to all at affordable prices;

(3) Act as catalyst to promote technological innovation and new applications;

(4) Promote seamless, interactive, user-driven operations of the NII;

(5) Ensure information security and network reliability;

(6) Improve management of the radio frequency spectrum;

(7) Protect intellectual property rights;

(8) Coordinate with other levels of government and with other nations;

(9) Provide access to government information and improve government procurement.

The HPCC Initiative is developing computing, communications and software technologies for the 
21 st century. To enable the NII initiative to build upon the HPCC Initiative, there is now a new component, Information Infrastructure Technology and Applications (IITA) [HPCC93] which explicitly addresses the third NII principle and implicitly addresses many of the others.

The IITA initiative supports the key areas of research and development and systems integration to demonstrate prototype solutions to National Challenges starting from the advanced technology level moving through the higher level of user capabilities to the ultimate user level in National Challenge projects. The IITA consists of four elements: (1) National Challenges (NC), (2) Information Infrastructure Services (IIS), (3) Intelligent Interfaces (II), and (4) System Development and Support Environments (SDSE). The latter three elements provide the NII support to enable the implementation of NII to national challenges. NIST is the government coordinating agency for the manufacturing applications National Challenge.

The government has formed an Information Infrastructure Technology Applications Task Group of the High Performance Computing, Communications and Information Technology (HPCCIT) subcommittee [ITA94] for the following purposes:

o Identify and document information technologies and their applications to national challenges;

o Identify and collect information about existing and proposed IITA activities within federal agencies participating in HPCC;

o Develop a plan for coordination of IITA activities among the agencies;

o Assist HPCCIT in reporting requirements related to IITA;

o Provide advice on matters of both technology and policy related to IITA to HPCCIT.

The Task Group is producing the following deliverables:

o Collection of National Challenge applications with an analysis of the infrastructure, services and developments needed. (A taxonomy of the National Challenges and associated information technology infrastructure has been produced [ITA94].) o Listing of federally sponsored FY'93 "ITTA-Related" projects;

o Listing of proposed "ITA-Funded" and "IITA-Related" work across participating federal agencies for FY'94 and FY'95.

The following subsections describe the taxonomy that was developed by the IITA task group. In Section 7, there is a listing of the FY 1993 NIST inventory of "IITA-Related" projects and a listing of the proposed work for all the federal agencies.

\subsection{National Challenges}

National Challenges are fundamental applications that have broad and direct impact on the Nation's well-being and competitiveness. The IITA program activity will support the use and integration of component technologies developed in other parts of HPCC to seek solutions for such applications. It will run the gamut of projects and programs ranging from concept demonstrations, experimental testbeds to the actual delivery of application systems in specific 
domains. The National Challenge applications will be used to demonstrate the generic information technologies that cross the applications.

Advanced Manufacturing - Supports work in advancing manufacturing technologies through the use of HPCC capabilities in design, production, planning \& quality control, marketing \& user services. A key element is the development of the infrastructure necessary to make the process and product information accessible over the information highway to both enterprises and customers. Research areas include concurrent engineering, protocols for electronic exchange of product data, electronic commerce for manufacturing, virtual design technologies, etc. The IITA focus will be on testbeds and technology demonstration on NREN.

Digital Libraries - Supports work in both technologies and applications which will lead to significant advances in the generation, storage and usage of digital information of different kinds across high speed networks. A digital library is a knowledge center without walls, open 24 hours a day and it is accessible via the network. Research areas range from advanced mass storage, on-line capture of multi-media data, intelligent filtering, knowledge navigation, effective user interfaces, system integration, and prototype \& technology demonstration.

Education. Training and Lifelong Learning - Supports work in making HPCC capabilities a major, universal resource for the nation's education, training, and learning systems at all levels and in all environments. Areas of work include innovations in providing network access and conducting pilot projects that demonstrate computing and communications technologies for improving learning/training that can scale up.

Health Care - Supports work in developing the concepts and technologies for applications of HPCC in the health sector. Such applications involve the use of high speed networking capabilities for linking health care resources of all kinds for research and shared care delivery. Novel use of emerging technologies, (e.g. visualization, virtual reality, 3-D imaging, multi-media databases, etc.) will be pursued.

Crisis Management - Refers to the use of command, control, communications, and intelligence information systems to support decision makers in anticipating threats, formulating plans, and executing these plans through a coordinated response. Supports work that deals with a variety of large-scale, time-critical, resource-limited problems, including nuclear monitoring, disaster operations, riot control, etc.

Energy Management - Supports work in managing the nation's energy resources - supply and demand - in an increasingly complex global environment. The IITA focus will be on the use of HPCC assets and capabilities to demonstrate ubiquitous benefits (e.g, energy savings) to the public from effective energy management.

Government Information Delivery Systems - Supports work in implementing new 
programs to develop and apply HPCC technologies to vastly improve public access to information generated by Federal, state, and local governments. Work include projects to connect agency depository libraries and other sources of government information to the Internet to enable public access; and to demonstrate, test, and evaluate technologies that will increase such access and effective use of government information.

Environmental Monitoring - Relates to our ability to observe, understand, and predict changes in the environment, both natural and man-made, on scales from seconds to millennia and from local to global. Efforts will focus on applying HPCC to advance environmental understanding and predictive capabilities, and on integrating multi-agency environmental observing systems (land-based, ocean-based, or satellite-borne) and historical databases using high-speed networks.

Electronic Commerce - Integrates communications, data management, and security services, to allow business applications within different organizations to automatically interchange information. Communications services transfer the information from the originator to the recipient. Data management services define the interchange format of the information. Security services authenticate the source of information, verify the integrity of the information received by the recipient, prevent disclosure of the information to unauthorized users, and verify that the information was received by the intended recipient. Electronic commerce applies and integrates these infrastructure services to support business and commercial applications, including financial transactions such as electronic bidding, ordering and payments, and exchange of digital product specifications and design data.

In August 1993, the Advanced Research Projects Agency (ARPA) hosted a workshop that investigated how the NII could be applied to two specific national challenges: manufacturing and education [ISAT93]. One important outcome of the workshop was the identification of general application requirements that would be needed to satisfy the vision for manufacturing and education of the future:

Plug and Play - Enables users to plug machines into networks and have them autoconfigured; enables system administration, diagnostics, and configuration management to be done remotely; and enables applications to determine level of network capability at the site, and possibly adapt delivery services accordingly.

Virtual Community - Allows for human to human interactions, whether synchronous or synchronous, organized on the basis of social relationships, directed tasks, or group membership.

Financial Transactions - Provides commercial transactions, including bidding, purchase orders, money, letters of credit and other electronic analogs of current financial transactions. 
Simulation/Virtual Reality/Remote Control - Provides the following capabilities: computer simulation of external objects and processes, and display of results; NII-supported simulation-engines, viewers, models and data that may be remote or real-time; hybrid simulations that may mix physical and virtual worlds; and on-line monitoring and control of remote equipment.

Object Management - Controls the process of storage, retrieval, communication, presentation, and replication of information objects. Provides a rich set of (decomposable) data types which interact across platforms.

Intellectual Property Management - Controls the use and disposition of intellectual property in electronic form. It is required for legal use of distributed or shared information.

Security and Privacy - Provides protection of the infrastructure and the rights of the user community.

Agents and Brokers - Allows for humans and computer processes which can be tasked to find, filter, and process information on behalf of users or processes. It simplifies the development and operations of large-scale systems through encapsulation.

Network Tools and Interface Conventions for Integrating Services - Provides canonical ways of hooking up services. It reduces the time and cost of developing new services by building on existing services.

The following three services represent the NII support for the National Challenges.

\subsection{Information Infrastructure Services (IIS)}

IIS provides the underlying network-capable building blocks upon which the National Challenges can be constructed. They will form the basis of the ubiquitous "Information Web" of the $21 \mathrm{st}$ century. Services bridge the gap between the communications bitways and the application-specific software components that implement the National Challenges. Services relate to domain design and searching and retrieving of data and objects; protocols, data fusion, exchange and integration; and databases and electronic libraries which include multi-media formats such as video, images, and audio. Security, privacy, and trusted systems issues extend into this area.

Universal Network Services - Includes techniques for improved ease-of-use, "plug and play" network interoperation, remote maintenance, exploitation of new "last mile" technologies like cable TV and wireless, management of hybrid/asymmetric network bandwidth, guaranteed quality of service for continuous media streams, and scale-up of network capabilities to dramatically larger numbers of users.

Integration and Translation Services - Provides a collection of services for migrating 
existing data files, databases, libraries, and programs to new better integrated models of computing, such as object-oriented systems. Techniques include "wrappers" which surround existing elements with new interfaces, integration frameworks which define application-specific common interfaces and data formats, and "mediators" which extend generic translation capabilities with more sophisticated knowledge-based computations.

System Software Services - Includes generic operating system services to support the National Challenges, including scalability across multiprocessors as well as networks of cooperating computers, geographic and administrative distribution of data and computational capabilities, real-time systems with support for guaranteed response time. and scalable storage, retrieval and I/O capabilities, including persistent storage, programming language support, and file systems.

Data and Knowledge Management Services - Includes extensions to existing database management technology for combining knowledge and expertise with data. Techniques include distributed databases; mechanisms for search, discovery, dissemination, and interchange; aggregating base data and programmed methods into "objects;" and support for persistent object stores incorporating data, rules, and computation.

Information Security Services - Provides support for the protection of the security of information, enhanced privacy and confidentiality for users of the infrastructure, the protection of intellectual property rights, and the authentication of information sources within the infrastructure. Techniques include privacy-enhanced mail, methods of encryption and key-escrow, and digital signatures, and authorization mechanisms and firewalls.

Reliable Computing and Communications Services - Provides the system software services for non-stop, highly reliable computer and communications systems that can operate continuously. Techniques include mechanisms for fast system restart such as process shadowing, reliable distributed transaction commit protocols, and event and data redo logging to keep data consistent and up-to-date in the face of system failures.

\subsection{System Development and Support Environments (SDSE)}

SDSE will provide the network-based software development tools and environments needed to build the advanced user interfaces and the information-intensive National Challenges themselves. It integrates elements of software development tools, frameworks and techniques to produce information services in virtual reality systems; collaboration software systems; applications specific templates and frameworks; software toolkits and generators; and automatic mappers and schedulers.

Rapid System Prototyping - Consists of software tools and methods that enable the use of incremental development and prototyping, focusing particularly on requirements engineering and systems design. Technologies include language support for evolutionary 
development of software components, specification techniques for complex software architectures, support for software systems with components written in multiple programming languages, and experimental tools to support the refinement of software prototypes into production quality systems.

Distributed Simulation and Synthetic Environments - Provides the specialized underlying support mechanisms for the creation of synthetic worlds, which can integrate real as well as virtual objects, both in terms of their visual as well as computational descriptions. Methods include distributed simulation algorithms, geometric models and data structures, tools for scene description, creation, and animation, and integration of geometric and computational models of behavior into an integrated system description.

Problem Solving and System Design Environments - Provides the techniques that support the software and system design process through the use of automated tools, with particular emphasis on maintaining flexibility and tailorability in tool configurations to enable organizations to tailor their support environments to their needs. Methods include efficient algorithms for searching huge planning spaces, more powerful and expressive representations of plans, operators, goals, and constraints, and the incorporation of efficient methods to facilitate scheduling and resource allocation.

Software Libraries and Composition Support - Supports the development of common architectures and interfaces to increase the potential for reusability across multiple underlying models of computation, the diversity of programming languages in use, and the varying degree of assurance provided by software components. Techniques include the development of the underlying methodology, data structures, data distribution concepts, operating system interfaces, synchronization features, language extensions, and other technology to enable the construction of scalable library frameworks.

Collaboration and Group Software - Supports group cooperative work environments. Methods include shared writing surfaces and "live boards", version and configuration management, support for process and task management, capture of design history and rationale, electronic multimedia design notebooks, network-based video conferencing support, and document exchange.

\subsection{Intelligent Interfaces (II)}

Advanced user interfaces will bridge the gaps between users and the future NII. A wide range of new technologies that adapt to human senses and abilities must be developed to provide more effective human-machine communications. The IITA program must achieve a high level user interface to satisfy the many different needs and preferences of vast numbers of citizens who interact with the NII. It includes intelligent user interfaces and advanced techniques for speech, text, written, and image understanding; knowledge based processing, multi-media computing and visualization; and high integrity, fault-tolerant and secure systems. 
Human-Computer Interface - Supports work in a broad range of technologies and their integration to allow humans and computers to interact effectively, efficiently, and naturally. Work in this area includes development of technologies for speech recognition and generation; graphical user interfaces that allow rapid browsing of large quantities of data; user-sensitive interfaces that customize and present information for particular levels of understanding; language understanding; multi-lingual machine translation; development of language and text corpora for experimental research; and human-machine interaction via touch, facial expression, gesture, etc.

Heterogeneous-Database Interfaces - Supports development of methodologies to integrate heterogeneously structured databases composed of multi-formatted data. Multi-formatted data may range from ASCII text, to numerical time series, to multi-dimensional measurements, to time series of digital imagery, etc.

Image Processing \& Computer Vision - Supports work in making images, graphics, and other visual information a more useful modality of human-computer communication. Research areas include all aspects of theory, models, algorithms, architectures, and experimental systems from low-level image processing to high-level computer vision. Methodologies of pattern recognition will be further developed to allow automated extraction of information from large databases; in particular, digital image data.

User-Centered Design Tools/Systems - Consists of work in models and methodologies leading to interactive tools and software systems for design and other user-centered activities. User-friendly tools that combine data-driven and knowledge-based capabilities is one of the areas for new research.

Virtual Reality and Telepresence - Supports work that will provide tools and methods for creating synthetic (virtual) environments to allow real-time, interactive human participation in the computing/communication loop. Such interaction may be through sensors, effectors, and other computational resources. The IITA focus is creating shared virtual environments which can be accessed and manipulated by many users at a distance in support of National Challenge Application areas.

\subsection{Advanced Manufacturing Technology Initiative}

The National Challenge being addressed by NIST is in manufacturing applications. There is another National Science and Technology Council (once called "Federal Coordinating Council for Science, Engineering, and Technology") initiative, Advanced Manufacturing Technology (AMT), which will help foster a shift in the way products are manufactured in the future, supporting the transition from rigid mass production to flexible manufacturing [AMT93]. The shift will be driven by major advances in underlying technologies such as computer hardware and software, and is made possible by wide-bandwidth communications, sensors, product and process design, and information processing. The manufacturing pilot project being proposed by NIST will build upon the technology being developed under the AMT initiative. 
There are four broad categories of advanced manufacturing: (1) Manufacturing Design Technologies - including tools and techniques used to prepare for manufacturing; (2) Manufacturing Technologies - including processes and equipment used for the actual production of physical products; (3) Supporting Technologies - including underlying core technologies needed to provide advances in design and manufacturing technologies; and (4) Manufacturing Infrastructure Elements - including concepts and mechanisms for managing the development of appropriate technologies and for encouraging their widespread use within the industrial base.

There are three areas identified as initial opportunities:

Intelligent Manufacturing Equipment and Systems - Incorporates "intelligent" sensors and advanced control techniques, innovative machine and tooling concepts, and seamless flow of product and production information. The goal is to develop, demonstrate, and promote industry deployment of the generic technology.

Integrated Tools for Product, Process, and Enterprise Design - Encompasses the operation of the entire manufacturing enterprise. Emphasis will be placed on providing an integrated framework, operating environment, common databases, and interface standards for a wide variety of emerging tools and techniques for designing manufacturing processes, equipment, and enterprises, as well as tools for evaluating the producibility of product designs.

Advanced Manufacturing Technology Infrastructure - Ensures that advanced technologies and concepts are understood and used by the private sector. Approaches may include: greater deployment of existing technologies; accelerated use of electronic techniques for transferring business and product information by small and medium-sized manufacturers; assistance in setting up education and training programs for shop floor workers, engineers, and management personnel; new mechanisms for monitoring international developments in manufacturing technology; and development of benchmarks that can be used to evaluate the effectiveness of manufacturing improvements.

There are several technologies and methodologies proposed in the AMT that can be used in accomplishing the objectives of the IITA manufacturing application. The intelligent manufacturing equipment and systems can be incorporated into the IITA manufacturing pilot project as the environment for which the NII must provide the "seamless web of communications networks, computers, databases, and consumer electronics." The integrated tools that are developed can be made available to the nation through various mechanisms developed under the NII. The advanced manufacturing technology infrastructure can be used by the customers for many of the services provided under the NII manufacturing applications element.

\section{DESCRIPTION OF NIST PROGRAM}

The program defined in this plan is part of a larger NIST program in the IITA initiative. The overall program can be divided into four major elements: (1) Systems Integration for 
Manufacturing Applications (SIMA), (2) Networking and Information Infrastructure, (3) Performance Measurement of Massively Parallel Processors, and (4) Advanced Software Technology and Algorithms. The first element deals with advanced manufacturing systems and networking that will be built upon the efforts of the other three elements which are concerned with the application of high performance computing and communications. The manufacturing application efforts are being performed by all the laboratories at NIST: Manufacturing Engineering (MEL), Electronics \& Electrical Engineering (EEEL), Physics (PL), Chemical Science \& Technology (CSTL), Material Science \& Engineering (MSEL), Building \& Fire Research (BFRL), Computer Systems (CSL), and Computing \& Applied Mathematics (CAML). The program will also establish a strong collaboration among the laboratories, both within the manufacturing and computing elements of the HPCC Initiative.

\subsection{Objective}

The primary objective of the SIMA program is to develop technologies for applications of highperformance computing and high-speed networking, with the first application designated as manufacturing. The effective utilization of these technologies can dramatically improve product quality and performance and reduce costs and time-to-market, thus greatly increasing American industry's ability to compete effectively in domestic and international markets.

Detailed objectives of the SIMA program are the following:

o Perform research and development in solutions to achieve integration of advanced manufacturing systems;

o Test high performance computers and networking hardware and software to determine their suitability to solve integration problems within the U.S. manufacturing community;

o Assist industry in the development and implementation of voluntary consensus standards;

o Serve as a demonstration site that industrial technology suppliers and users can use to identify and overcome technical and other barriers leading to the successful and costeffective implementation of advanced manufacturing systems and networks;

o Provide training for industry and technology deployment agents on the effective use of these new technologies;

o Provide mechanisms for information exchange.

\subsection{Rationale for SIMA Program}

Producing today's complex products through the principles of concurrent engineering requires the ability to store and retrieve product and process data far beyond our existing capabilities. New 
data interface standards and supporting technologies must be developed for this new data sharing capability to be successful. Furthermore, technologies are needed to enable the development and implementation of production systems for monitoring both system performance and product quality throughout the life-cycle. For example, major advances in the technology of intelligent controls, knowledge databases, sensory processing, and communication tools are essential to aid the transition to concurrent engineering.

The theme of the program is that the development of effective systems integration technologies is necessary if the U.S. manufacturing companies are to take complete advantage of the NII and the supporting information technologies. Technology innovation is not the only requirement to meet the vision for making the NII a reality for advanced manufacturing. Standards are needed to facilitate economic interactions that require a high level of cooperation and coordination. Standards also help to determine the efficiency and effectiveness of the economy as well as the cost, quality and availability of products.

However, governments are using standards to protect their domestic markets and to develop new international ones, to the detriment of U.S. trade. In particular, the harmonization of European standards, which was completed in 1993, will not only make it harder for U.S. companies to trade in Europe, but will allow the Europeans to take the lead in setting international standards.

Standards have become more important due to our growing reliance on technology. The growing pace of technological change also drives the need for standards. The faster the advance of technologies, the greater the risk in $\mathrm{R} \& \mathrm{D}$ and product development. In fact, it is now necessary to develop standards even before the actual products are made available to the customer.

In the 21 st century, the concept of developing technical standards before the commercialization of the associated products will take hold of the U.S. industrial community. Technology advances will occur so rapidly that previous standards activities can not keep up with the demand for technical standards. The world community will realize the importance of objective technical standards such as those embodied in product data exchange standards and enterprise frameworks and will put more pressure on the international standards efforts to approve standards quickly so that international concurrent engineering process needs can be met.

The report "Global Standards: Building Blocks for the Future" [OTA92] focuses on how U.S standards and standards development processes might affect U.S. trade. There are many questions addressed in the report but the one of particular interest is "whether and to what extent does the U.S. standards process support the growth and competitiveness of the U.S. economy in a rapidly changing global environment." There were several findings of importance to the concept of standards and competitiveness:

o A growing national stake in standards issues;

o Need for greater attention to standards;

o Need for cooperation rather than conflict among U.S. standards bodies;

o Need to strike a more appropriate balance between the public and private sectors. 
o Inadequate federal coordination and policy making;

o Need for greater attention to how other governments use standards to create markets for their nations' industries;

The report also describes how the U.S. and other countries are rapidly evolving into informationbased, knowledge societies, where the creation, use, and communication of information plays a central role. It is more and more being treated as a commodity to be bought and sold in the marketplace. This trend will greatly affect standards development. Information can be used to improve the overall efficiency of the production process itself. This growing importance is evident from the continued growth of the information sector of the economy.

Flexible Computer-Integrated Manufacturing with all its associated technologies has clearly been identified as a critical technology in making U.S. industry more competitive internationally. Standards have also been identified as key enablers in the development of the "virtual enterprise" which allows for the broad base of U.S. companies to work together in producing world class products. The question is "why hasn't industry been more involved in adopting standards in their activities?"

One reason why industry has been slow to adopt standards is because of the slowness in which the standards community can react to approving standards through the consensus process. Information technology is evolving so rapidly that companies prefer to purchase the latest products in the belief that they will provide a technology edge over their competitors. Obviously these products are not based on standards that can be approved in an acceptable time frame.

The answer to this problem is to develop new approaches to the implementation of standards that significantly accelerate the standards approval process. Program initiatives need to be developed that analyze the life cycle development of standards and implement tools and procedures that accelerate the availability of standards. The future will involve the concurrent development of new technology and corresponding standards that allow vendors to build products to meet the technology needs. This work will be a major focus for this program.

However there are several issues that must be addressed in order to implement the data interface standards needed to effectively integrate manufacturing enterprises:

o Manufacturing applications environments today are company specific;

o Applications are in a constant state of flux as new technologies are implemented;

o Standards that are applied are either proprietary or company limited. For this reason, systems integration remains an art;

o Cost of integration within government manufacturing facilities alone is presently $\$ 4 \mathrm{~B}$ and growing; 
o Although large companies can fund internal integration efforts, the cost of integration is keeping small businesses from participating in the virtual enterprise operation.

Standards can in themselves be thought of as products. Using the world class definition of a product, the following requirements must be achieved:

o Broad-based - consensus across industrial community;

o Timely - keeping up with changing technology;

o Quality - correctness;

o Cost-effective - economical to implement and cost effective to produce;

o Functional - reflect the requirements of the applications.

The basic premise is that systems integration technologies can reduce the time to design and manufacture a product. There are three steps to developing the necessary integration technology solutions. The first step is to develop an understanding of the information content of the applications. The next step is to use data modeling techniques in integrating the applications. The final critical step is to propose standards as the enabler of integration between companies. There have been many efforts, both industry and academic, directed at the first two steps. However it is the third step which involves reaching a consensus on the integration framework that is the most difficult and time consuming and cannot be properly addressed by individual organizations.

\subsection{Proposed Program}

The purpose of the Systems Integration for Manufacturing Applications (SIMA) program is to create a collaborative multi-year technology development program involving NIST, U.S. industry, universities, and other government agencies in order to develop, refine, test, and transfer advanced, computer-integrated, electronically-networked manufacturing technologies and associated applications. Through the SIMA program, current research and development will be expanded in such areas within flexible computer-integrated manufacturing (FCIM) as product data exchange (PDE) and electronic data interchange (EDI) that will allow NIST to perform its appropriate role as a Commerce agency for research and development in standards and technologies.

The role for NIST includes the following types of activities:

o Produce the methodologies for developing standards;

o Produce the testing methods and services;

o Lead national/international standards efforts as appropriate;

o Provide "custodian of the standard" function;

o Develop prototype standards as appropriate;

o Implement prototype manufacturing systems that demonstrate the effectiveness of potential standards;

o Coordinate HPCC manufacturing applications development throughout the government; 
o Demonstrate and disseminate HPCC technology;

o Provide reliable data for quality technical decisions through the standard reference data program;

The program contains four elements: (1) Manufacturing Systems Environment (MSE), (2) Technology Transfer Environment (TTE), (3) Standards Development Environment (SDE), and (4) Advanced Manufacturing Systems and Networking Testbed (AMSANT) Facility.

The Manufacturing Systems Environment (MSE) is the element in which the research and development of advanced manufacturing systems and networking will be performed. Typical products from this environment will be prototype systems that reflect the enhancements from information technology, interface specifications for the manufacturing applications that can be forwarded to standards organizations, and technical papers.

The Technology Transfer Environment (TTE) is the element in which the results of the MSE efforts as well as other efforts in the United States in the area of advanced manufacturing systems and networking can be transferred to American industry. Typical products of this environment will be workshops, training materials, accessible electronic data repositories, and pre-commercial prototype systems that can be installed by potential vendors in potential user facilities for test and evaluation.

The Standards Development Environment (SDE) is the element that will support the development of standards within SIMA. Through this environment, a major standards-based data exchange effort will be targeted at flexible computer-integrated manufacturing to alleviate the lack of data exchange capability among computer-aided activities [e.g. Computer-aided design (CAD), Computer-aided process planning (CAPP), and Computer aided manufacturing (CAM)]. It is also important to make available metrics, performance measures, and testing methodologies for commercial implementations.

The AMSANT facility is the element that serves as the operating laboratory at NIST with extensive computing, networking and manufacturing capabilities. It will serve as the major laboratory resource for the projects developed under the three SIMA program environments. The facility will be used by the NIST technical staff, industry and other government agencies to perform research, to develop prototype systems, and to develop performance measures for advanced manufacturing applications defined by the MSE program. The SDE projects will utilize the computing hardware and software tools of the AMSANT facility to develop and test proposed manufacturing systems and data exchange standards, and to develop methods for verifying conformance of manufacturing systems to the emerging standards. The facility will also serve as a primary focal point for collaboration activities defined by the TTE.

\subsection{Five Year Outlook}

Over the first five years of the initiative, prototype facilities will be constructed that will demonstrate to companies how systems integration technologies can be effectively utilized for 
concurrent engineering practices. During the first two years, an integrated reference model for the prototype manufacturing systems will be developed and appropriate systems installed and modified to implement the model. By the third year, a conformance testing service will be available so that manufacturing application vendors will be able to market systems that conform to the standards defined for systems integration. By the fifth year, standards for the use of shared database technology with manufacturing applications will be available that will allow for the effective use of concurrent engineering methods. The research will also produce reference model architectures, metrics, and performance measures for advanced manufacturing processes and networks.

Indicators of success in carrying out the above program elements are:

- Technical quality and quantity of advanced computer-integrated, electronically-networked, manufacturing technologies developed in the SIMA program and demonstrated in the AMSANT facility;

o Number of pre-production prototype products successfully installed by potential vendors in potential user facilities;

o Number of pre-commercial products that find their way into the marketplace, and the dollar value of sales of such products;

o Number of draft standards produced and number of proposed standards tested and evaluated;

o Amount of industrial consortia participation in the Advanced Technology Program as evidenced by proposals submitted in areas related to the SIMA program;

o Ability of industry to utilize the results of this program; including large corporations, teaming partners, and the small and medium-sized manufacturing supplier base (through participation with existing and new Manufacturing Technology Centers as they are created);

o Amount of interaction with other government agencies by supporting their missionspecific technology and standards requirements;

o Amount of participation by universities and other educational institutions in research activities and the establishment of training channels addressing both management and technological education for advanced manufacturing systems and networking;

o Amount of support for national and international standards activities;

o Capability to provide information dissemination in key technology areas; 
o Acknowledgement of the American workforce that both the quality of the work environment and the quality of the product has been significantly enhanced by SIMA program staff;

\section{PRODUCT DATA STANDARDS AS KEY}

The program is built upon the premise that interface standards are necessary to make systems integration an effective tool for advanced manufacturing systems. The Standard for Exchange of Product Model Data (STEP) is considered to be the key standard for the integration activities, although there are many other interface standards that will be part of the program.

\subsection{Importance of Product Data Exchange Standards}

Emerging technologies can influence the entire manufacturing process. As an eample, consider how the mechanical drawing has impacted the way industrial processes have been performed, and how a new technology for managing product data will enable concurrent engineering to revolutionize U.S. manufacturing.

Before the dawn of the industrial revolution, engineering work was defined by a physical model of a product to be reproduced. For example, a worker manufacturing a rifle barrel would ensure that the dimensions of the barrel corresponded to a model barrel by using calipers to transfer measurements from one to the other. This method reinforced the concept of workers manufacturing specific product types rather than generic components of larger products.

In 1801, Gaspard Monge wrote "La Geometrie Descriptive" as the first treatise on modern engineering drawings. This included the theory of projecting views of an object onto three planes and the addition of size specifications to the shape descriptions. With the mechanical drawing an objective standard of performance for workmanship was possible and thus the model couid be eliminated. The drawing enabled the practice of designing a product with interchangeable parts to be created. Operations could then be performed using contractors that could manufacture different pieces to be assembled. This capability led to the fragmentation of the manufacturing process which exists to this day.

The mechanical drawing concept has lasted for almost 200 years. As described above, the manufacturing process for developing quality products was interwoven with the method for describing the products. The drawing became the output of the design process and the input into the manufacturing process. Drawings were converted into process plans which were converted into programs or procedures for the manufacturing operations. Thus every process has its own view of the product data. These dissimilar views have made it difficult to feed back knowledge about different processes to the designer. In today's industrial enterprises, the life-cycle processes for a product are no longer all performed by the same group of people. In fact, the processes are distributed through a network of factories.

As we move into the twenty-first century, new manufacturing technologies are needed to improve 
productivity and competitiveness. In this information and computer age, companies exchange and share information across the country. This capability is needed for manufacturing the complex products such as automobiles, airplanes, ships, and buildings that are produced today. There is a special consideration for accelerating this information exchange process since the existing products and technologies are often replaced before their useful life has expired as manufacturers compete in the marketplace. To meet production deadlines, computer-aided design tools are used to move products from concept, through design, prototyping, manufacturing, testing and, ultimately the support that is required by the customer.

Multi-enterprise concurrent engineering will require the ability to store and retrieve product data far beyond the capability of the mechanical drawing. The replacement for the mechanical drawing that will allow the new engineering technologies to be implemented in a revolutionary manner is Product Data Sharing. This new capability will make available to the designer knowledge about all other processes. It will process product data through automated computerbased techniques that allow for shared access among the life-cycle processes in support of concurrent engineering. It will make available an integrated product data model that allows for the multiple views of the product to be accessed. New data interface standards and supporting technologies must be developed for this new product data sharing capability to be successful.

The ability to quickly and efficiently share product data information among a variety of different computing environments is critical to any collaborative effort either within a company or across different companies cooperating in normal business and commerce. The representation of product data in digital form is a new technology which is basic to both a company's internal plans for integration and its external relationships with the world. Product data exchange is the key component of the NIST program to implement the standards and technologies required to make the NII applicable for manufacturing.

The term product data denotes the totality of data elements which completely define a product for all applications over its expected life-cycle. Product data includes the geometry, topology, tolerances, relationships, attributes and features necessary to completely define a component part or an assembly of parts for the purposes of design, analysis, manufacture, test, inspection and product support. The product model is expected to be informationally complete for purposes such as generating manufacturing process instructions, directing quality control testing and performing product support functions.

\subsection{Background on STEP}

The importance of this new technology has been recognized by CAD/CAM users throughout the industrialized world and has given impetus to an international project coordinated through the International Organization for Standardization (ISO) to develop a single world standard for product data exchange.

Unanimous agreement exists on the need for creating a single worldwide standard which enables the capture of information to represent a computerized product model in a neutral form without 
loss of completeness and integrity, throughout the life-cycle of the product. Known internationally as STEP and domestically as PDES (Product Data Exchange using STEP), the project focuses on the representation and exchange of product models with sufficient information content as to be interpretable directly by advanced application computer programs with little need for human interaction.

Internationally, the development has been led by the ISO committee on Manufacturing Languages and Industrial Data (TC184/SC4) chaired by NIST. Their work is called STEP, formally known as ISO 10303. The U.S. effort has been centered in the NIST-led IGES/PDES Organization project called PDES. Overall U.S. coordination is performed by the National Initiative for Product Data Exchange hosted by NIST and staffed by industry, universities and federal agencies. The key U.S. development efforts in STEP technology are led by PDES, Inc., a consortium of over twenty U.S. (and two British) technology companies with the specific goal of accelerating the development and implementation of STEP, and the NIST National PDES Testbed, a government effort (supported jointly by Department of Commerce and the Department of Defense) to assist in the development of the testing methods and supporting technologies.

Excellent international cooperation has produced good progress toward developing and implementing the standard. The ISO committee TC184/SC4 has become the world focus for the development of digital product data technology, and it has attracted a capable team of international experts. By unanimous vote of ten countries, the ISO committee decided to register the STEP Initial Release as a Draft International Standard. The vote was taken on February 26, 1993, in Turin, Italy, and the action capped a major technical collaboration of over 300 experts across 16 countries who worked together to produce the 2300 pages of standards documentation.

The Initial Release of STEP is complete in that it validates all concepts of the STEP technology architecture including information modeling, formal data specification language, conformance testing methodology and the application protocols (which contains the application specifications needed by vendors to implement products).

The Initial Release addresses two priority application areas, drafting and product configuration management. Users have already recognized the need for other applications with work already well underway on twenty-three additional applications that will be added to STEP in the near future. These span a wide range from shipbuilding to electrotechnical plants, from sheet metal dies to automotive design, from electronics to composites, and from NC machining planning to dimensional inspection planning.

The vote by the SC4 committee highlights a broad technical consensus across Europe, the US and Japan that the Initial Release of STEP is now technically complete and that widespread commercialization of this important technology can now proceed. The decision by SC4 gives a "green light" to multiple implementation efforts worldwide at both vendor companies and at five STEP Centers in France, Germany, Japan, the UK and the US.

The STEP effort is expected to accelerate the evolution of concurrent engineering, support 
Electronic Data Interchange (EDI) electronic commerce and enable business partners to share sophisticated digital product data as easily as paper drawings are shared today. As such, the STEP project promises to be one of the most influential standards that has ever been developed in the industrial automation field.

\subsection{STEP Technology}

An important part of supporting the standards efforts is to provide the methodologies and tools for application protocol development. "Application protocols (APs)" convert end-user requirements into specifications that can be used to test conformance of vendor implemented application software (i.e. configuration control of design data) to the standard. The APs define the scope (using an activity model), the information to be exchanged (using an information model), the means of testing (using test suites), and a users guide for implementing the application.

In the case of STEP, APs have been developed using the following supporting parts that are also part of the overall standard. There are a collection of resource models which contain a generic set of basic product information entities (such as tolerance, geometry, shape, material, drafting, kinematics, etc.) that are used to develop the emerging APs. There are also a set of Methods which define the means in which APs will be tested (e.g., conformance), implemented (e.g., file exchange, shared database), described (e.g., using an object-oriented information modeling language EXPRESS) and constrained.

Application Protocols will be implemented that apply across a wide range of products such as mechanical, electronics \& electrical, apparel, distribution systems, and structures (e.g., for shipbuilding and construction.) The long term goal is to develop sets of application protocols that will be complete for a given type of product in a given application such as design or manufacturing. These applications allow for the testing of the proposed standards and to further understanding and improvements in the standards and methods. It is through the development of the applications and relevant application protocols that technology can be implemented and then transferred to industry.

\section{PROJECT DESCRIPTIONS}

This section describes the projects that fall within the four SIMA program elements. The SIMA program involves the participation of all eight NIST intramural laboratories: Manufacturing Engineering Laboratory (MEL), Electronics \& Electrical Engineering Laboratory (EEEL), Building \& Fire Research Laboratory (BFRL), Chemical Science \& Technology Laboratory (CSTL), Computer Systems Laboratory (CSL), Computing \& Applied Mathematics Laboratory (CAML), Materials Science \& Engineering Laboratory (MSEL), and Physics Laboratory (PL). The MEL, BFRL and EEEL are primarily involved in addressing the industrial technology needs. The CSL and CAML are involved in providing support in the HPCC core technology. The PL, CSTL and MSEL are primarily involved in addressing the scientific and engineering reference data requirements for manufacturing. The specific laboratories will be indicated for each of the 
projects described in this section.

A typical technology project within the SIMA program will proceed through the following phases:

o Define requirements and develop an understanding of technology;

o Develop specifications for system(s) representing the technology;

o Implement a prototype system that augments the present system with the required interface specifications;

o Transfer the prototype to other facilities (e.g. AMSANT facilities);

o Work with the Advanced Technology Program to get industrial involvement in implementing new technology;

o Facilitate the development of a standard based on the earlier specifications;

o Develop conformance testing procedures for vendor implementations;

o Provide training and information dissemination of the technology, making use of the Manufacturing Technology Centers, as appropriate.

This section describes projects that will be initiated in FY 1994 and supplemental projects to be initiated in FY 1995 that will be in addition to the FY 1994 level of funding.

\subsection{Manufacturing Systems Environment}

This element involves the research and development of technology and standards in support of a broad range of industrial manufacturing domains that include mechanical products, apparel, electronics, construction, and chemical processing. The major thrust is in the development of the technologies for integrating manufacturing systems that support each of these manufacturing domains. In addition there are efforts that address the need for developing critical scientific and engineering data required for use in advanced manufacturing systems.

\subsubsection{Objective}

The objectives of the Manufacturing Systems Environment (MSE) are:

o Perform research \& development into integration technologies and advanced manufacturing systems;

o Develop and test prototype advanced manufacturing systems that can be demonstrated 
as integratable across a network;

o Develop and test integration solutions for design, planning, and production software applications within a manufacturing product life cycle, where these applications are accessible across a communications network at distributed sites;

o Incorporate HPCC technology into systems integration solutions;

o Make HPCC an integral part of the design, planning, and production of manufactured products and demonstrate the increased manufacturing capability.

\subsubsection{Technology Areas}

The taxonomy of advanced manufacturing systems technologies created for the Advanced Manufacturing Initiative will be used as the primary basis for identifying the scope of the technology areas to be considered for the MSE part of the SIMA program.

The MSE advanced manufacturing technology areas will be characterized into two main thrust areas - Advanced Manufacturing Systems (AMS), and Manufacturing Integration Technologies (MIT). The AMS research and development activities focus efforts on typical application and systems areas such as design (product, process, and enterprise), planning, scheduling, process modeling, shop control, simulation, inspection, assembly, and machining. The MIT activities focus research on integration technologies and interface requirements to support integration of manufacturing applications and systems across and between multiple enterprises. Typical integration and interface technologies include networking, communications, database technologies, frameworks, and information exchange for product and process data.

Other technology areas that may be needed in order to effectively develop and test the advanced manufacturing systems include (1) Machine tool, Robot and Coordinate Measurement Machine (CMM) interfaces, and (2) Real-time control architectures and operating systems.

\section{1:3 Major Activities}

The major activity of MSE will be the development of interface specifications for advanced manufacturing systems. MSE will develop and analyze product models, including models for electromechanical systems, product dynamics, and life cycle product simulations. The projects will support the development of prototype process models for use throughout the manufacturing environment. As opportunities arise, the element will spin off other NIST services and products, including standard reference data, measurement services, and other applications.

\subsubsection{Expectations}

In the long term, the MSE projects will include research and development activities to develop the following: 
o New design tools, methods, and algorithms for rapidly developing high-quality product designs incorporating advanced technology;

o Technology to extend and apply design to the entire product life cycle;

o Novel design techniques, including reconfigurability and upgradability;

o Modular, flexible, reconfigurable, and affordable production processes;

o New methods to obtain and share information quickly, and to link information directly to production processes;

o Seamless networking in the industrial base utilizing network protocols to transfer data between customers and contractors;

o Specifications for enterprise product model data bases;

o Demonstrations of Application Protocols required for specific processes (such as design and process planning);

o Production control environment with control and manufacturing systems of the AMSANT facility and specifications for enterprise production control data bases.

In addition, the following specific methods will be pursued:

o Promote wide use of standard product data that are fully implemented at all levels of design and manufacturing;

o Make available manufacturing knowledge and computers able to simulate and model the entire manufacturing process;

o Apply design, systems integration architectures, and product data to shop floor control, including collaborative design, knowledge base concepts for engineering design and manufacturing, use of design tools, and next-generation controllers.

\subsubsection{Projects to be Initiated for FY 1994}

There are ten projects under the category of Manufacturing Systems Environment. The first six projects are related to systems integration needs for the specific industrial sector. Four of these projects - Control Systems Architecture (MEL), Integration Specifications (MEL), Design Applications (MEL), and Planning Applications (MEL) - are concerned with developing the integration framework for mechanical parts production. The fifth project, Process Plant Engineering and Construction (BFRL), is concerned with the construction industry. The sixth project, Interoperability of Chemical Measurements and Data Systems (CSTL), is concerned with 
the chemical instrumentation industry. A seventh project, Electronic Commerce for the Electronics Industry (EEEL), will develop prototype demonstrations showing how on-line journals can be used as an intelligent index to technical component information and can serve as an entry point for on-line commerce. An eighth project, Virtual Design Technology (CAML), is in support of developing a specific HPCC enhancement for manufacturing of mechanical parts. A ninth project, Packaging and Interconnect Materials Supporting HPCC Infra-Technology (MSEL), relates to developing improved materials data needed for manufacturing electronic components. The tenth project, Collaborative Environment Platform (CSL), is concerned with providing an improved software platform in which to develop the systems integration technologies.

\section{Control Systems Architecture}

The objectives of this project are: (1) build an integrated reference model architecture for intelligent production control covering all levels of control from motor drives to an entire factory, (2) demonstrate the architecture in a specific discrete parts manufacturing application, (3) write guidelines for implementing the architecture, and (4) build engineering tools for implementing the architecture. The architecture project will build on previous work in control systems architectures done by the Robot Systems Division and the Factory Automation Systems Division within the Manufacturing Engineering Laboratory. The scope of an architecture may be specified in three independent dimensions - domain, life cycle, and organizational extent. The domain of this architecture is the control of mechanical systems for discrete parts manufacturing. The portion of the life cycle on which the project will focus is manufacturing. The organizational extent will include manufacturing planning, scheduling, and production.

\section{Systems Integration}

This project will advance the state of the art of integration mechanisms and information models as applied to the domain of mechanical product design, analysis, and manufacturing planning. The goal is to demonstrate the seamless exchange of information between manufacturing applications that are also interfaced to a shared database environment. This project will integrate one or more client applications with two or more server applications. The integration mechanism will be an object request broker. This project will establish a partnership with the design and planning applications projects in order to establish specifications for integrating these applications.

\section{Design Applications}

The focus of this project is aimed at addressing issues related to "Design for Produceability" (DFP) which includes linking "Design for Assembly" (DFA) tools with existing and emerging Computer-Aided Design (CAD) applications, and how to effectively apply HPCC technology. DFP is defined as the utilization of information and knowledge from all business and engineering areas that are impacted by the design of a product. This explicitly acknowledges that DFP crosses many domains and covers a broad area including all other targeted design activities such as "Design for Manufacturability" and "Design for Cost". Due to this large scope, the FY 1994 
efforts will be limited to Design for Manufacturing and Assembly (DFM/A) elements. This project identifies and addresses issues that restrict the acceptance of DFM/A solutions. The objective is to identify and develop approaches that remove the restrictions of the applications of DFA tools and methods.

\section{Planning Applications}

The focus of this project is on improving the capabilities and integration of manufacturing planning applications. This will be addressed in three ways: (1) specification of a series of information models and communication protocols needed by planning systems, (2) implementation of these specifications as part of an integration activity using commercial planning system software, and (3) development of a repository of information supporting the research, development and integration of future planning system applications.

\section{Process Plant Engineering and Construction}

This project will adopt emerging virtual manufacturing technologies to allow process plant engineers to design for constructability, and to allow the planning of the use of construction automation on the plant site. As an initial focus, the project will consider the structural systems which support process plant equipment and piping systems. Additional systems will be included as the research progresses.

\section{Interoperability of Chemical Measurements and Data Systems}

The project will provide for interoperability of chemical measurement systems to facilitate the development of automated chemical processes that involve quantitative chemical analysis. It will also provide for compatibility and interoperability of reference chemical data, data produced in chemical measurements and software used for the processing and interpretation of chemical data. The key element of the project is defined by CAALS-I, which is a first level messaging protocol between chemical measurement devices/instrumentation and controllers.

\section{Electronic Commerce for the Electronics Industry}

This project will work with industry and other government agencies to provide prototype demonstrations and level 1 services via the NII that would benefit the electronics industry. NIST will lead a demonstration team developing an Electronic Business Reply Card (EBRC), showing how on-line journals can be used as an intelligent index to technical component information and can serve as an entry point for on-line commerce. Other demonstration scenarios to be developed include the development of a CAD interface to allow engineers to search for and procure electronics component information in a computer-sensible format. NIST will provide a multiplatform testbed for demonstration development and coordination.

Virtual Design Technology 
The virtual design approach will span experimental efforts based on the design of experiments and Taguchi methods, process modeling involving control system theory, continuous mathematical models including optimization technologies, discrete methods such as computational geometry, economic techniques for design and investment decisions, and computer simulation experiments. The resulting computer based prototype will be realized within a high performance computing network of workstations for virtual engineering design.

\section{Packaging and Interconnect Materials Supporting HPCC Infra-Technology}

The project focuses on packaging and interconnect issues above the chip and active optoelectronic element levels. Technical activities are directed towards processing, inspection methods, properties data, performance measurement and improved materials for two major components of electronic and opto-electronic packaging and interconnects: substrate materials \& encapsulants and conductors.

\section{Collaborative Environment Platform}

The project will develop a HPCC integrated platform which demonstrates on-line visualization, interactive data manipulation, audio and video conferencing technologies for access to multiple data sets in collaborative environments and interactive scientific analysis applications. This platform will demonstrate to engineering and manufacturing organizations the ability to use currently available products for accomplishing remote collaboration. In addition, this demonstration platform will give the context to look into the data storage problems associated with the use of large volumes of data in both wideband communication networks and LANs.

\subsubsection{Supplemental Projects Proposed for FY 1995}

There are seventeen projects proposed under the category of Manufacturing Systems Environment. Eight of these projects - Control Systems Architecture (MEL), Integration Specifications (MEL), Design Applications (MEL), Planning Applications (MEL), Interoperability for Chemical Process Measurements and Data Systems (CSTL), Electronic Commerce for the Electronics Industry (EEEL), Virtual Design Technology (CAML), and Collaborative Environment Platform (CSL) - represent extensions to projects initiated in FY 1994. The eight new projects proposed under MSE will address different manufacturing domains and computing applications that encompass the product life-cycle.

Three additional projects that will be introduced by MEL in FY 1995 include Manufacturing Systems Engineering Tools, Advanced Apparel Manufacturing Systems, and Electronic Commerce for Mechanical Parts Manufacturing. The first project will place an emphasis on providing an integrated framework, operating environment, common databases, and interface standards for a wide variety of emerging software tools and techniques for designing manufacturing processes, equipment; and enterprises, as well as tools for evaluating the producability of product designs. The second project will address the development of apparel design and production software within the MEL AMSANT in order to implement integration 
solutions as well as prototype new apparel design engineering techniques for apparel manufacturing. The third project will address the harmonization between the electronic commerce applications and the manufacturing applications along with the associate information.

A new BFRL project, Process Plant Conceptual Design, deals with the development of systems integration technologies for the process plant industry. It is a complementary project to the FY 1994 project in Process Plant Engineering and Construction. Another new BRFL project, Building Systems Design and Operation, will develop an integrated model for the performance of buildings and their systems.

A new EEEL project, Application Protocols for Electronic Products, deals with the development of systems integration technologies for the electronics Industry. Another two new MSEL projects deal with the development of improved use of materials information in manufacturing systems: Open-Systems Manufacturing of Enabling Materials and Integration of Materials Performance Data into Computer-Aided Design. Finally there is a new project in the standard reference data area that will be addressed by the Physics Laboratory - Integration of Reference Data Systems into Information Networks.

\section{Manufacturing Systems Engineering Tools (MSET)}

The major objectives of this project are: (1) identify key manufacturing systems engineering methodologies, (2) develop a prototype manufacturing systems engineering workstation, (3) specify and promote needed MSET architectures and standards, and (4) enable the implementation of remote on-line accessible manufacturing systems engineering computing services. In keeping with the theme of systems integration, this project will address several integration problems within the mechanical parts manufacturing community. These problems include: (1) lack of interoperability of current factory engineering software tools, (2) lack of commercial availability or sufficient functionality of many needed engineering tools, and (3) lack of a clear system specification or demonstration of the power of such an integrated computing environment.

\section{Advanced Apparel Manufacturing Systems (AAMS)}

The goal of this project is to apply systems integration specifications and develop practical standards that will impact the footwear and apparel industries. An advanced manufacturing systems environment will be implemented to integrate the pre-production activities necessary for the manufacture of shoes and clothing. An information infrastructure that can bring consumers closer to the manufacturer and enable manufacturers to produce and distribute products more efficiently is necessary in order to reverse the decline of the US based apparel manufacturing industry. The pre-production applications that will be addressed include design, planning, scheduling and control. 


\section{Electronic Commerce for Mechanical Parts Manufacturing (ECMPM)}

The project will address the development of the technology and standards required to conduct electronic commerce for mechanical parts manufacturing. Systems such as procurement, inventory management, supplier bidding, accounting, etc. will be analyzed for their information requirements. The manufacturing systems will be extended to interface with these business systems to build an overall enterprise environment. The manufacturing information that overlaps the business information must be melded together using common data modeling techniques so that manufacturing enterprises will be able to have a "seamless" interface to both types of information. A common information architecture will be developed, along with a common implementation mechanism using a shared database approach. This project will be closely coordinated with the project "Harmonization of Standards" which addresses STEP and EDI harmonization.

\section{Electronic Commerce for the Electronics Industry}

This project will develop a consensus vision and architecture for electronic commerce appropriate to the requirements of the electronics community, and will coordinate the development of enabling tools and protocols that are deemed to be missing. NIST will leverage the lessons learned from the Electronic Commerce of Component Information (ECCI) Electronic Business Reply Card (EBRC) demonstrations to draft an architecture appropriate to the broader needs of the electronics industry for network-based commerce. NIST will work closely with those companies developing enabling tools to ensure that solutions are created which include small businesses as well as large enterprises.

\section{Process Plant Conceptual Design}

This project will develop, refine, test, and transfer integration technologies and interface specifications needed by the U.S. process plant industries to introduce concurrent engineering practices using high-performance computing and communications in the conceptual design of process plants. The project involves developing: (1) A reference model for comprehensive process plant information bases, (2) An integration strategy for project definition, cost estimating, and task planning and scheduling in process plants, and (3) Information models that account for design and for design task management. The information models will be demonstrated through the use of trial STEP application protocols. The reference model, information models, interface specifications, and trial application protocols developed in this project will be implemented through the establishment of national and international consensus standards.

\section{Building Systems Design and Operation}

This project will develop an integrated model for the performance of buildings (including but not restricted to buildings in process plants) and their systems, such as mechanical, environmental, and fire protection. New concurrent engineering practices based on this model that can optimize building designs against performance requirements will be explored and documented. New 
building operation and control techniques based on this model that can optimize actual performance against expected and unexpected factors will similarly be explored and documented. This project builds on expertise gained in past research at NIST and through liaisons with professional engineering societies and industry. The results will be implemented through the establishment of national and international consensus standards.

\section{Application Protocols for Electronic Products}

In response to the needs expressed by the Initial Graphics Exchange Specification (IGES) community, this project will develop an IGES Application Protocol for layered electrical products. The work will be based on the hybrid work published as NIST Technical Note 1295 , as well as the layered electrical product model written by the Cal Poly Task Team. A team will be formed with industry and other national labs to ensure a consensus standard is produced. In following years, this AP will be brought into STEP, and work on other priority electrical/electronics STEP AP's will be developed through the international umbrella of IEC TC93.

\section{Open-Systems Manufacturing of Enabling Materials}

This project aims to create a systems integrated virtual-manufacturing test-bed suitable for exchange across data superhighways among materials suppliers, manufacturers and technology incorporators to demonstrate just-in-time advanced materials manufacturing. The NIST program to develop and demonstrate this test-bed will allow the exchange of materials manufacturing data, improve the competitive capabilities of the U.S. advanced materials manufacturing industry, facilitate the insertion of advanced materials into already existing products and new applications, and support industry-identified needs for advanced materials application and commercialization.

\section{Integration of Materials Performance Data Into Computer-Aided Design}

The project will develop prototype systems integrating material performance information into $\mathrm{CAD}$ tools and performance analysis software. The technical focus will be industrial problems such as corrosion and fracture avoidance in the design of real-time process control equipment operated in corrosive environments at remote sites. The work will use existing standards for sharing information and will also identify the need for further standards development.

\section{Integration of Reference Data Systems into Information Networks}

This project will establish a network interface to a NIST digital library of critically evaluated atomic, molecular, and optical reference data, which will allow public access by remote users using industry-standard protocols. The system will accept "expert-access" questions regarding the data, the questions being prioritized by a scientific administrator for transmission to NIST laboratory staff. Network access to the digital library will be made available via industrystandard routes: anonymous ftp, electronic mail, and usenet newsgroups. 



\subsection{Technology Transfer Environment}

\subsubsection{Objective}

The objectives of the Technology Transfer Environment (TTE) are:

o Develop technology transfer infrastructure which can be used to exchange manufacturing information using HPCC technology;

o Develop prototype information services in collaboration with industry partners which could become commercialized products;

o Develop services related to document searches and retrieval of government and other research reports relating to: manufacturing, electronic journal access \& on-line video manuals, and teleconferencing \& distributed collaborative work environments;

o Establish communication channels for network of researchers and implementors of manufacturing technologies;

o Facilitate technical workshops and executive seminars;

o Facilitate technology demonstrations.

\subsubsection{Technology Transfer Strategy}

The strategy for implementing a technology transfer program will include the following:

o Develop road map and strategic plan for transferring HPCC technology to manufacturing applications;

o Define state-of-the-art with respect to on-line knowledge bases, identify near term advances, and determine opportunities for deploying HPCC into mainstream manufacturing operations;

o Involve both companies which develop manufacturing systems and those which use these systems;

o Develop pre-commercial prototype systems and work with vendors to install them in user facilities for test and evaluation;

o Provide training for industry on the effective use of new technologies;

o Work with private industry to develop requirements for standards for the use of advanced computer-based training systems, including multimedia and interactive learning 
technologies that will accelerate the efficient use of advanced manufacturing systems;

o Provide mechanisms for the exchange of information about advanced manufacturing systems and networking;

o Assist universities and other educational institutions in expanding their manufacturing engineering programs.

\subsubsection{Activities}

The SIMA Technology Transfer Environment will provide:

o Applications of HPCC technologies;

o Technical workshops;

o Training courses and material;

o Technology demonstrations;

o Executive seminars;

o On-line access to documentation (AMSANT specification and studies) and software (pre-commercial proof of concept systems);

o External program coordination;

o Communication channels for network of researchers and implementors of AMSANT technologies.

\subsubsection{Expectations}

In the long run, the technology transfer environment will produce the following capabilities:

o Electronic library service for the STEP standard and STEP public domain software;

o National repository for knowledge bases associated with manufacturing systems.;

o AMSANT linked into electronically networked learning centers and teaching factories;

o Groupware for requirements definition and analysis added to the AMSANT services;

o Additional on-line libraries of publications and software allowing users direct access to the SIMA deliverables.;

o Shared databases, email and advanced video conferencing techniques enabling the number of AMSANT participants to grow across the United States. Geographically distributed development and implementation teams formed as a virtual enterprise for the advancement of manufacturing engineering technologies.; 
o SIMA training programs using interactive and multi-media learning systems.

\subsubsection{Projects to be Initiated for FY 1994}

There is only one project to be initiated in FY 1994: Manufacturing Information Technology Transfer (MEL).

\section{Manufacturing Information Technology Transfer}

The project will define and implement a set of software tools and services for disseminating SIMA and HPCC technology information, and related research results and information exchange standards between NIST and industry collaborators. Communication channels will be established for a network of researchers and implementors of manufacturing technologies through the NIST AMSANT.

\subsubsection{Supplemental Projects Proposed for FY 1995}

There are three projects proposed under the category of Technology Transfer Environment: Manufacturing Information Technology Transfer for Mechanical/Apparel Products Industry (MEL), Technology Transfer of HPCC Information into the Electronics Industry (EEEL), and Technology Transfer for the Construction Industry (BFRL). The former is an extension to the technology transfer project initiated in FY 1994.

\section{Manufacturing Information Technology Transfer for Mechanical \& Apparel Products Industry}

The project will establish a manufacturing engineering education outreach program that includes liaison with major universities, extending the cooperative education program, sponsorship of $\mathrm{PhD}$ candidates, and participation of guest professors. The activities will be strongly linked to the development of the National Research and Education Network (NREN) to enable manufacturers to take advantage of numerous information-based services, such as ready access to supplier information, product catalogues, remote training, and participation in electronic competitive bidding. A long term strategic technology transfer plan which includes the use of HPCC technology to improve government laboratory interactions with all types of manufacturing industries will be developed during FY 1995. This strategic plan will be developed from a series of workshops between commercial systems developers, industry users of HPCC technology, and a committee representing NIST and other government laboratories.

\section{Technology Transfer of HPCC Information into the Electronics Industry}

This project will set up a program to assist U.S. electronics companies in the rapid adoption of the HPCC information infrastructure technology in their business operations. This program will disseminate information on standards and promote the use of standards as a mechanism for creating a competitive advantage for a business. The infusion of productivity enhancing standards into the U.S. business culture will be done principally through education. This will be 
accomplished by distributing periodic updates of documentation on the status of the standards and by holding seminars and tutorials on the standards.

\section{Technology Transfer for the Construction Industry}

This project will build on and add to the emerging technology transfer environment to assist the U.S. construction industry in the dissemination of critical technological information including codes and standards, materials and product specifications, and performance assessment methodologies. Potentially thousands of requirements apply to any given construction project throughout its life. New technology transfer mechanisms are urgently needed to ensure that project participants can locate, access, and, especially, use correct information in a timely fashion. Liaison will be established with appropriate codes and standards organizations that serve the U.S. construction industry. Standards and guidelines will be developed as required by the industry to implement these new technology transfer mechanisms.

\subsection{Standards Development Environment}

\subsubsection{Objective}

The objectives of the Standards Development Environment (SDE) are:

- Assist industry in implementing voluntary consensus standards relevant to computer integrated manufacturing (CIM), including standards for networking, electronic data interchange, and digital product data sharing;

o Facilitate industry efforts to develop and test new applications of advanced manufacturing systems and networks;

o Facilitate efforts to develop and test new data exchange standards utilizing HPCC technology;

o Accelerate industry deployment of consensus standards.

\subsubsection{Technology Areas}

The following are typical technical support areas for developing standards:

o Conformance testing - Perform activities that test the capability of a vendor-developed product to conform to an existing standard;

o Application Protocol Development Environment - Provide an environment of tools and information that facilitate the creation of application protocols by standards developers;

o Standards Documents Repository - Provide an orderly framework within which the 
development of standards can take place. NIST can serve as the "Holder of the Standard";

o Information Modeling - Provide a mechanism for the representation of the information required for specifying the standard;

o Standards Methodologies - Provide mechanisms for the development of methodologies to be used within the standards development process that lead to the implementation of quality standards;

o Manufacturing Software Quality Assurance - Provide mechanisms for testing manufacturing software to determine if it is performing the correct operations.

\subsubsection{Activities}

The following are typical activities to be performed in the SDE:

o Work with standards developers to facilitate development of application protocols;

o Maintain configuration management of standards documents;

o Develop toolkits for standards processes;

o Perform qualification, integration, and editing of evolving standards;

o Develop new information modeling techniques in support of standards specifications;

o Perform conformance testing of vendor products;

o Serve on standards committees;

o Chair national and international standards organizations;

o Develop better standards development practices.

\subsubsection{Expectations}

In the long term, the SDE will

o Demonstrate new levels of standards development environment;

o Oversee the implementation of application protocols needed for the product life-cycle process;

o Publish conformance testing procedures for product data exchange and FCIM applications;

o Finalize harmonization with existing standards in information technology;

o Make recommendations on manufacturing data interface standards implementations based on demonstrated performance; 
o Refine FCIM specifications and submit to appropriate standards bodies;

o "Systematize" the entire spectrum of standards, the testing and evaluation methods, and the supporting technologies in a definitive report on CIM Interface Standards Evaluation \& Technology.

\subsubsection{Projects to be Initiated for FY 1994}

There are six projects initiated in FY 1994. Three of the projects - Conformance Testing for STEP (MEL), Application Protocol Development Environment (MEL), and STEP for the Process Plant Industries (BFRL) - relate to the development of interface standards required for systems integration. The project, Application Interface Development Environment (CSL), relates to the development of tools that will enable information to be exchanged across the data highway. Two other projects - High-Speed Electronic and Optical Components (EEEL), and Video Processing (EEEL) - relate to the development of improved measurement capability for selected HPCC electronic components.

\section{Conformance Testing for STEP}

There are two major elements to this project: (1) conformance testing system development and (2) the conformance testing program. In the first element, STEP-based testing tools and test suites for specific application protocols will be developed. In the second element, methods for product certification, test system validation, and test laboratory accreditation will be defined. The success of these elements will be evaluated in trials with early implementations of the first STEP application protocols. Once the technical basis for STEP conformance testing has been demonstrated, the focus will be on the automation of the test execution and analysis and on the delivery of testing services. This technology will be integrated into software product development environments which leverage existing Computer-aided Software Engineering (CASE) environments. The technology will then be transferred to testing laboratories to provide permanent test services, and commercialized for distribution to vendors and users.

\section{Application Protocol Development Environment}

This project will establish an integrated suite of software tools to assist application protocol developers to create, test, and implement product life-cycle application interface specifications. The environment will promote the development of high-quality specifications while at the same time decrease the overall cost of producing the specifications. These specifications, known as APs, are the implemented portions of STEP. Suppliers of computer-aided design, manufacturing, or logistic systems will be evaluated for conformance to specific STEP APs and will be required to exchange product data according to such protocols. AP specifications submitted for international standardization must meet special criteria for industry need, content, and quality. Such specifications typically require extensive rework as they progress through the standards process. Current practices used for AP development incur extraordinary labor expenditures on behalf of AP developers to define the requisite components of an AP and to deliver them as a 
finished AP document. While AP developers may use some software tools to help them accomplish their AP development tasks, the tools work independently, are not integrated, and have not been customized specifically for the purposes of AP development.

\section{STEP for the Process Plant Industries}

This project will assist the United States process plant industries in developing the application protocols needed to implement concurrent engineering applications, shared integrated product databases, and construction automation practices using STEP. Specific issues to be addressed include (1) the selection and use of commodity items, (2) the development of process and instrumentation (P\&I) diagrams from process stream information, (3) the development of piping system geometry from P\&I information, (4) the capture of project management data, (5) demonstrating compliance with the ISO 9000 quality standards, and (6) the exchange of piping system data from designer to fabricator. NIST will build prototype implementations of key application protocols in both integrated database and file exchange environments for testing and demonstration purposes.

\section{Application Interface Development Environment}

This project will assist industry in the development of both product and process interface standards that will enable information to be exchanged across the data highway. An application interface development environment will be implemented that will demonstrate the capability of making the tools necessary for the development and testing of interface standards available to the industrial community and support the integration of standards for specific applications. The initial challenge will be to design the application interface development environment by addressing the specific tools necessary for the development and testing of data management, graphics and imaging standards.

\section{High-Speed Electronic and Optical Components}

At present U.S. industry is experiencing a major shortfall in the measurement capability required to develop optical and electronic components, in both individual and integrated forms, for highspeed computer networks. An important part of these needs relates to the efficiency of signal transfer at optical and electronic interconnections. These interconnections are weak links in highspeed systems. This project will provide improved measurement support for industrial development of better optical interconnections and electronic interconnections between electronic integrated circuits operating at gigabit-per-second speeds.

\section{Video Processing}

The power to exchange image information among application areas and across geographic boundaries, to display it once received, and to measure the impairments which occur in the transmission and display process will expand the utility of that data and the number of its potential users. The development of standards for interoperable video systems and of 
measurement practices for image fidelity and quality are necessary precursors to the implementation of advanced communication systems. The project will develop generalized measurement techniques, i.e. video quality metrics, for image fidelity and quality for video information.

\subsubsection{Supplemental Projects Proposed for FY 1995}

There are six projects proposed for the Standards Development Environment. There is a general theme of providing effective support environments for the development of standards as well as facilitating the harmonization across the broad spectrum of standards that compose the range of information and supporting technology required for enterprise integration. Several projects will be involved in coordinating the support mechanisms and information across the various industries. Three are extensions of projects proposed in FY 1994: Application Protocol Development Environment (MEL), Conformance Testing for STEP (MEL), and Application Interface Development Environment (CSL). A new project within MEL, Harmonization of Standards, will address the problems involved in the effective integration of manufacturing information standards. Two projects - Infrastructure for Interoperable Electrical \& Electronic Product Data Exchange Standards (EEEL), and Concurrent Engineering of Information Exchange Standards (EEEL) - deal with supporting the development of standards for the electronics industry. Another project, Software Production and Integration Environment (CSL), deals with software development as a product itself.

\section{Harmonization of Standards}

An effort will be initiated that will begin the harmonization between the manufacturing data interface standard STEP and the business electronic exchange standard EDI (Electronic Data Interchange). The manufacturing information that overlaps the business information must be melded together using common data modeling techniques so that manufacturing enterprises will be able to have a "seamless" interface to both types of information. This project will be tied together with "Electronic Commerce for Mechanical Parts Manufacturing." The harmonization effort will be coordinated within both of the concerned standards organizations.

\section{Infrastructure for Interoperable Electrical/Electronic Product Data Exchange Standards}

Integral to the electronics industry are product standards and specifications that enable the design, manufacture, documentation, procurement, and support of modern electronics. Among the technical challenges is the development of adequate information models that describe the essential characteristics of electrical and electronic products. Currently, there are at least four established standards which can be used to transfer data among automated tools for fabricating electronic products. This project will work to create an overarching system to accommodate the current standards, and will support harmonization efforts through the creation of enabling tools, such as an on-line data dictionary and active'data interpretation programs capable of appropriate data conversion. 
This project will study the needs of the standards development process and develop infrastructure tools to support the concurrent development of information exchange standards. Problems, such as the absence of a mechanism for intra-committee and inter-committee issue tracking and inability to quickly and cost effectively distribute drafts of documents to pertinent parties, slow the process of developing standards and reduce the quality of standards that are produced. The standards development process will continue to be a bottleneck for the information infrastructure and it will be difficult or impossible to create the complex standards needed to support highly automated systems engineering tools without improving the existing standards development process. This project is intended to provide a standards-development architecture that will prevent future "harmonization" problems among existing electrical/electronic exchange standards.

\section{$\underline{\text { Software Production and Integration Environment }}$}

The products covered by the initial set of STEP Parts (components of the standard) are primarily mechanical in nature. This project focuses on computer software as an essential component in the products themselves as well as in manufacturing process. The project will investigate how high-quality software can be developed in a concurrent engineering environment, tested, integrated with other components of a product, maintained, and reused in other products. The initial challenge will be to prototype a software repository of commercial and research Integrated Software Engineering Environments (ISEE) tools within the STEP environment on several different platforms using existing networks. This prototype will be used to test and validate the framework of STEP standards and procedures for software production and integration.

\subsection{AMSANT Facility}

\subsubsection{Objectives}

The objectives of the AMSANT facility are:

o Serve as a demonstration site that industrial technology suppliers and users can utilize as well as serve as a proof-of-concept for the research and development activities at NIST.

o Serve as the interface to a network of technology development testbeds within the United States that are selected through the Advanced Technology Program to develop, refine, test, and transfer advanced manufacturing and networking technologies and associated applications.

o Serve as the interface to one or more information dissemination contacts (defined among the NIST Manufacturing Technology Centers) for the purpose of providing information and technical assistance regarding advanced manufacturing and networking technologies to small- and medium-sized manufacturers. 


\subsubsection{Architecture}

A computing systems architecture will be designed and developed to support a wide range of requirements for projects supported by the AMSANT program. The testbed architecture will contain the most advanced networking capabilities, communication tools and interface standards available in today's market.

\subsubsection{Activities}

The initial phase of the NIST AMSANT facility will support a limited set of R\&D activities and standards testing for MEL and BFRL projects. In follow-on phases, it is expected that the AMSANT facility will also support activities of other NIST laboratories working on HPCC research. Specifically, the AMSANT facility seeks to support the following range of activities:

o Developing and testing interface specifications for manufacturing systems;

o Testing and comparing communication systems for industrial collaborator;.

o Serving as a conduit of information on issues and advancements in manufacturingrelated technologies;

o Providing capabilities to demonstrate and showcase research results and standards development;

o Serving as a mechanism for bringing internal NIST projects together to provide a laboratory-wide resource for manufacturing research;

o Performing evaluations and state-of-the-art assessments in architecture design for integrating production planning and control environments.

\subsubsection{Expectations}

The AMSANT will be a virtual "living" laboratory environment, distributed across the NIST organizations participating in the SIMA program. It will include a distributed set of computing workstations located throughout NIST dedicated to the research activities of the program. MEL and BRFL will establish physical testbed locations within each laboratory in order to support future demonstration of HPCC technology, and support a wide range of research activities. The MEL and BRFL testbeds will be established early in the program to provide demonstration environments for MSE related projects. New technologies and facilities will be incorporated as the course of the program is charted so that the testbed is at the technological forefront and is fully responsive to the needs of the program and U.S. industry. 
The testbed will:

o Provide communications and networking capabilities for nationwide collaboration with industry, academic and other government agencies;

o Acquire and install a series of state-of-the-art computer-based manufacturing systems for engineering and production research projects;

o Set up an emulated production control environment with existing commercial systems, in order to develop and test new interface specifications developed by project in the MSE;

o Demonstrate the standards development environment;

o Initiate the use of application protocols for SIMA applications;

o Publish a facility architecture document defining the major components and capabilities.

The testbed will build upon other facilities available at NIST such as (1) Rapid Response Manufacturing, (2) Process Planning Testbed, (3) Manufacturing Design Testbed, (4) National PDES Testbed, (5) Algorithm Testing Service, (6) Automated Manufacturing Research Facility, (7) National Advanced Manufacturing Testbed, and (8) a variety of robotics testbeds.

\subsubsection{Projects to be Initiated for FY 1994}

There are three projects for FY 1994: AMSANT for Mechanical Parts Production (MEL), Operator Interfaces For AMSANT (MEL), and AMSANT for Process Plant Industries (BFRL).

\section{AMSANT for Mechanical Parts Production}

The AMSANT technologies will include advanced networking and communications, high performance computing, and advanced manufacturing applications to support R\&D activities by NIST researchers. The facility will provide communications and networking capabilities for nationwide collaboration with industry, academic and other government agencies. The facility will include a series of state-of-the-art computer-based manufacturing systems for engineering and production research projects. The facility is also expected to have available an emulated production control environment with existing commercial systems, in order to develop and test new interface specifications for manufacturing systems. In addition,

\section{Operator Interfaces for AMSANT}

This project is part of the AMSANT facility for Mechanical Parts Production. The project will implement and demonstrate an operator interface system that will allow human operators to 
visualize what is going-on anywhere in the manufacturing facility, to diagnose and correct problems, and to control (within appropriate limits) any process or machines. A variety of display technologies will be developed that can give the operator of software systems a choice of windows into the system's operation. One approach is to generate graphics images that allow the operator to visualize the functional condition of the machines or processes of interest. View point control can allow the operator to "fly" through the facility and visually observe the movement of machines and materials. Advanced forms of such displays might incorporate "virtual reality" techniques with 3-D and holographic displays, goggles, helmets, etc. Either real or simulated facilities can be displayed. These technologies will be evaluated in order to determine their benefits to support manufacturing applications.

\section{AMSANT for Process Plant Industries}

The objective of this project is to establish an experimental computing and communication testbed in which to (1) establish the industrial baseline in process plant engineering and construction, (2) serve as proof-of-concept for the process plant testbed for integration activities of industrial partners in the process plant activity, and (3) serve as a communications hub for all United States participants. This testbed will be a distributed component of the NISTwide AMSANT. In addition to the generic resources provided throughout NIST, this component of the AMSANT specifically will support advanced software applications dealing with process plant design, procurement, construction, and operation, and high performance databases.

\subsubsection{Projects Proposed for FY 1995}

There is only one new project proposed under the category of AMSANT - AMSANT for Apparel Production (MEL). The MEL and BFRL FY 1994 AMSANT projects will be extended in FY 1995.

\section{AMSANT for Apparel Production}

The AMSANT for Apparel Production follows the objectives of the overall AMSANT project. The testbed will include a series of state-of-the-art computer based apparel manufacturing systems for engineering and production research projects. The testbed is also expected to have available an emulated production control environment with existing commercial systems, in order to develop and test new interface specifications for apparel manufacturing systems.

The expanded projects in Mechanical Parts Production and Process Plants will develop an emulated manufacturing systems environment supported by high performance computers, and connected to the national data highway. The emulated environment will be used to develop and test interface standards and information models for integrating design and manufacturing systems in the mechanical and apparel manufacturing industries. State-of-the-art computer-aided design systems, combined with planning, scheduling and control systems will be implemented in the AMSANT, and integrated by a complete and unambiguous product and process data model. The NIST AMSANT will be connected to, and communicating with the industry supported 
AMSANTS, other government laboratories, and industrial collaborators, by the national data highway. AMSANT will offer a complete infrastructure of software, hardware, and networked resources to enable a full range of collaborative work among scientists.

\subsection{Interactions with Industry}

Each of the NIST laboratories have existing interactions with industry in the projects defined in this plan. It is expected that these interactions will be expanded and new interactions formed. This section lists the interactions by laboratory.

\section{Manufacturing Engineering Laboratory}

The Manufacturing Systems Environment program is expected to work closely with industrial research staff and commercial systems developers to assemble and extend a suite of software systems that reflects the current state-of-the-art. The program will interact with a number of external partners including National Center for Manufacturing Science (NCMS) Rapid Response Manufacturing (RRM) Consortium, Consortium for Advance Manufacturing International (CAMI), American Society for Mechanical Engineering (ASME), and the American Apparel Manufacturers Association (AAMA).

The Technology Transfer Environment program is expected to actively recruit new guest workers from industry and academia, and pursue Cooperative Research and Development Agreements (CRDAs) with industry sponsored AMSANT's. The program will work closely with industrial research staff and commercial systems developers to transfer development results directly to manufacturing facilities.

The Standards Development Environment program. will work closely with the IGES/PDES Organization (IPO), ANSI CIM Standards Board, International Standards Organization Technical Committee on Industrial Automation (TC184), PDES Inc., the National Initiative for Product Data Exchange (NIPDE) and the Continuous Acquisition and Life-Cycle Support (CALS) Program.

The AMSANT facility will be used by both NIST researchers and industry collaborators to test integration concepts, incorporate standards, and ensure the success of implementing HPCC technologies in manufacturing systems. It is expected to work closely with the new industrial testbeds, and collaborate with other government agencies by establishing new CRADA'S.

\section{Materials Science and Engineering Laboratory}

The Packaging and Interconnect Materials Supporting HPCC Infra-technology project has been developed with input from the electronics and opto-electronics industries gained during separate NIST-sponsored industry-led workshops held in May 1990 and August 1992. The program will build upon existing projects in electronic packaging materials and expand upon present cooperation between NIST and the Semiconductor Research Corporation (SRC), the Semiconductor Industry Association (SIA), Microelectronics and Computer Technology 
Corporation (MCC), and numerous individual firms. Outputs from this program will be translated through industry partners and leveraged through consortia and industry associations such as SRC or OIDA.

The Open-systems Manufacturing of Enabling Materials project will build upon its already existing cooperation with the U.S. transportation industry - chiefly the Automotive Composites Consortium and the Aerospace Industry Association - and the U.S. advanced materials industry namely the Advanced Composites Materials Association - for the implementation of this project.

The Integration of Materials Performance Data into Computer-aided Design project will build upon the work over the past decade where NIST has developed a unique set of materials databases and expert systems for metals and ceramics. This work has been done in partnership with industry. Partners have included technical and professional societies, individual companies, industrial consortia, and information vendors. These partners will be involved in the proposed work.

\section{Building and Fire Research Laboratory}

The Process Plant Engineering and Construction project will establish liaison with existing organizations such as the Construction Industry Institute and the Stanford Center for Integrated Facility Engineering to ensure that its research is coordinated with industry activity. The CRDA program will allow direct interaction with specific industry players.

The STEP for the Process Industry project will create a Process Industry Action Group that will provide input and feedback on industry needs for STEP. NIST will work collaboratively with existing organizations including the American Institute of Chemical Engineer's Product Data Exchange Institute (PDXI), the Construction Industry Institute (CII) and its Construction Industry Action Group (CIAG), the Navy-Industry Digital Data Exchange Standards Committee (NIDDESC), the American Petroleum Institute's Petroleum Industry Data Exchange (PIDX), and the Petroleum Open Software Consortium (POSC). NIST will establish liaison with the U.K. Process Industry STEP Consortium, and the ESPRIT CAESAR and ProcessBase projects. The CRDA program will enable direct interaction with specific companies.

The AMSANT Facility for Process Plants project will work closely with commercial vendors such as CADCentre, Intergraph, ComputerVision, Auto-trol, and ASG, whose software is used widely in the process industry, and with engineer/constructor companies such as Bechtel, Stone and Webster, Fluor Daniel, and others, who have developed in-house software systems to deal with process plant design and construction.

The Process Plant Conceptual Design project will build upon existing efforts. The Process Industry Action Group established in FY 1994 will be expanded as needed to include planners and designers. NIST will continue its collaboration with such organizations as the American Institute of Chemical Engineers Product Data Exchange Institute (PDXI), the Construction Industry Institute (CII) and its Construction Industry Action Group (CIAG), and the American 
Petroleum Institute's Petroleum Industry Data Exchange (PIDX). NIST will continue its liaison with the ESPRIT CAESAR and ProcessBase projects. NIST will seek collaborative programs with plant owners such as Du Pont and Tennessee-Eastman and engineering/construction companies such as Bechtel, Stone and Webster, and Fluor Daniel. The CRDA program will enable direct interaction with specific companies.

\section{Computing \& Applied Mathematics Laboratory}

Industrial collaboration is essential to the success of the Virtual Design Technology project. Industry will be involved in planning through workshops. In the execution of research, industrial expertise will be incorporated through the on-going successful NSF/ASA/NIST Research Fellows program and through other guest researcher arrangements. Opportunities to make use of CRDA's will be developed.

\section{Chemical Science \& Technology Laboratory}

The Interoperability of Chemical Measurements and Data Systems project will work closely with industry. Communications standards will be developed through the workshops associated with the NIST Consortium on Automated Analytical Laboratory Systems which includes major industrial users such as DuPont, and Eastman Kodak; and instrument manufacturers such as Hewlett-Packard and Perkin-Elmer. Collaborations are being initiated with chemical industrial representatives from such companies as Dow Chemical, and Aldrich Chemical to adapt current software for their internal use. Collaborations will be developed with the ASTM Subcommittee E-49.52 and the Analytical Instrument Association to develop common nomenclature in chemical measurement systems.

\section{Computer Systems Laboratory}

The Software Production and Integration Environment project will work closely with national and international standards organizations, STEP related organizations, developers and users of ISEE tools, vendors of products with embedded software, and government programs in defense conversion, manufacturing, and enterprise integration.

The Application Interface Development Environment project will work closely with national and international standards organizations, developers and users of automated design and development tools, and large and small businesses who will be developing implementations of standards and using standard-conforming products.

\section{Electronics \& Electrical Engineering Laboratory}

The High Speed Electronic and Optical Components project has been developed in consultation with industry and will be carried out similarly. The Telecommunications Industry Association (TIA) has helped in identifying industry's measurement needs and will assist in organizing the round-robin measurement intercomparison for FY 1994. TIA members will participate in that 
round robin. The Institute of Electrical and Electronics Engineers has sponsored conferences and produced publications that have aided NIST in defining electronic-circuit interconnection measurement problems. NIST is working with a committee of the Electronic Industries Association focused on interconnection problems. One CRDA with industry is in place and another is under development to address interconnection characterization. An engineer with relevant industrial experience has been asked to join this project as a post-doctoral fellow. Measurement capability developed by this project will be incorporated in voluntary industry standards as appropriate.

The Video Processing project will work with industrial and governmental laboratories, e.g. NTIA and David Sarnoff Research Center, and with standards organizations, e.g. SMPTE, in developing and evaluating our models and tests.

The Electronic Commerce for the Electronics Industry project is currently collaborating with the following agencies, consortia and companies to build successful ECCI demonstrations: Allied Signal, Boeing, CAD Framework Initiative, CADIS, Cahner's CAPS, EE Times, Honeywell, INFO Enterprises, Lawrence Livermore National Laboratories, Navy RAMP, NASA, Mentor Graphics, Microelectronics and Computer Technology Corporation (MCC), National, Sandia National Laboratories, TacTech, Technology Decision Resources, Texas Instruments, and the University of Utah. NASA is currently supplying an engineer to NIST's AEMP testbed to work on electronic commerce tasks.

Success will also require a liaison with the following standard organizations which have a vital interest in component parts and classification schemes: IEC TC93, IEC TC3D, ISO WG2 Part Libraries, and CFI Component Information Representation (CIR) subcommittee.

The Technology Transfer of HPCC Information into the Electronics Industry project is a key element in the transfer of standards technology from NIST to industry. EEEL will work with the regional Manufacturing Technology Centers in the development and deployment of the HPCC information infrastructure technology to U.S. industry.

The Infrastructure for Interoperable Electrical/Electronic Product Data Exchange Standards project has already been approached by the EDIF steering committee to provide a neutral site for industry to have standards-based products conformance tested. NIST will work closely with consortia, like PDES, Inc. and agencies such as Navy RAMP, to demonstrate standards interoperability.

The Concurrent Engineering of Information Exchange Standards project will work with industry to help create the PDES Migration Guide. NIST will coordinate work with NASA, Sandia Laboratory, LLNL, Smart Valley, and the NIPDE ECCI project.

\section{Physics Laboratory}

The Integration of Reference Data Systems into Information Networks project will consult and 
work with industrial users at all stages of this project. Appropriate workshops will involve representatives of industry and standards organizations in the planning and implementation of the digital library. We will also take any opportunity to obtain industrial expertise through the NSF/ASA/NIST Research Fellows program and the use of CRDA's.

\section{DELIV ERA BLES}

This section describes the deliverables for the SIMA program. The program will be developing over four years. In FY 1994, the program has been approved for funding at the $\$ 9 \mathrm{M}$ level. The program funding for FY 1995 is planned with an increase of $\$ 16.2 \mathrm{M}$ to reach the $\$ 25.2 \mathrm{M}$ level (this is the level presently in the President's budget to Congress). There are planned increments for FY 1996 and FY 1997 which would set the final level of the program at approximately $\$ 35 \mathrm{M}$. There are four types of deliverables discussed: (1) high-level set of program deliverables over the life of the program (assumes the merger of SIMA initiatives from FY 1994 to FY 1997), (2) generic deliverables to be produced each year, (3) deliverables for the next five years for those projects initiated in FY 1994, and (4) deliverables for those projects initiated in FY 1995.

\subsection{Long Tem Deliverables}

The following are long term deliverables for the Advanced Manufacturing application subelement within the National Challenges element of the IITA program. The SIMA program uses the same set of deliverables (assumes that new initiatives are begun each year up to FY 1998 and brings in expected collaboration with other agencies):

\section{Install Applications: FY 1994 - FY 1998}

M1. Establish and augment advanced manufacturing systems and networking testbeds (AMSANT) throughout some government agencies that perform manufacturing research as well as with agency contractors who are building systems to support agency functional requirements. This will demonstrate to industry how systems integration can be effectively utilized to allow for such strategies as Agile Manufacturing and Concurrent Engineering to be applied across enterprise boundaries. Select sets of manufacturing applications based on current industry needs and assist with the installation of state-of-the-art systems. Establish links to other testbeds within industry and academia to help evaluate, measure, and disseminate effects of new technology in order to demonstrate "virtual" AMSANTs. Provide on-line capability for industry to access manufacturing technology information using such systems as Xmosiac and World Wide Web.

\section{Develop Interface Specifications: FY 1994 - FY 1997}

M2. Develop interface specifications for the set of manufacturing applications chosen in M1 that will be used to implement an integrated manufacturing environment. Publish these specifications as proposed standards in such areas as product data exchange and interoperation of heterogeneous databases and applications. Choose applications that extend across the manufacturing life-cycle for a given industry (e.g., mechanical part production) as well as across industries (i.e., 
electronics, process plant design). Publish frameworks for the integration of the standards required for the harmonization of the applications involved in the implementation of both the manufacturing and information technologies.

\section{Extend Applications: FY 1995 - FY 1998}

M3. Based on interface specifications from M2 and applications installed from M1, build and support the development of application interfaces (APIs) so that the applications can easily adopt these extensions. Demonstrate the integration of applications across local and NII networks that link multiple AMSANTs. Begin exploring the concept of "brokering" tools from different vendors to built integrated tools for concurrent engineering tasks/time-specific use (e.g integrate different types of simulation and analysis tools that are needed for requirements during design, manufacturing, distribution, maintenance and recycling).

\section{Develop Conformance Testing: FY 1996 - FY 1998}

M4. Develop, evaluate, and publish conformance testing methods and services to assess vendor conformance to the proposed specifications (and emerging standards) developed in M2. Support the participation of small businesses and academics in the standardization process. Demonstrate an advanced selection of prototype vendor products that conform to the specifications. Implement mechanisms and systems that accelerate the development of the critical standards needed for manufacturing integration.

\section{Implement Database Environment: FY 1997 - FY 1998}

M5. Implement an interoperating, heterogeneous manufacturing database environment to support the applications implemented under M3. Develop an integrated database for product and process information required for sets of manufacturing applications. Publish specifications for the industry/application database. Demonstrate that this environment can communicate among and co-exist at multiple sites.

\section{Demonstrate Virtual Enterprise: FY 1998 - FY 1999}

M6. Demonstrate the fully integrated set of applications that access both product and process information through the manufacturing database, using both large and small industrial sites. Specifically provide demonstrations illustrating (1) the ability for customers to "custom design" products, (2) agile manufacturing among enterprises, (3) supplier-contractor interactions such as bidding systems, (4) concurrent engineering for product development, and (5) enterprise integration including both advanced manufacturing and electronic commerce applications.

\subsection{Generic Deliverables}

The following deliverables are generic to all the projects and programs within SIMA. They will be initially generated in FY 1994 and updated each year. 
o Publish a requirements document (based on prior research and development) for the use of HPCC for advanced manufacturing systems.

o Publish a technical development plan that defines the overall Systems Integration for Manufacturing Applications (SIMA) program.

\section{Manufacturing Systems Environment}

o State-of-the-practice papers published on applications in advanced manufacturing systems.

o State-of-the-art papers published on applications in advanced manufacturing systems.

o Manufacturing applications software (e.g., process planning, scheduling, computer-aided design) installed and functioning.

o Interface specification of application data available.

\section{Technology Transfer}

o Workshop proceedings published.

- Implementation plan for nationwide collaboration published (includes technology transfer mechanisms and application of HPCC capabilities for technology transfer).

o On-line access capability (developed by HPCC) provided to reports and papers generated in program.

\section{Standards Development}

- Plan published that identifies overall standards requirements for advanced manufacturing.

o Plan published that identifies specifications for the environment needed to support the standards requirements for advanced manufacturing.

\section{AMSANT Facility}

o Requirements document published for AMSANT.

o Demonstration of installed and functioning AMSANT. 


\subsection{Five Year Deliverables for Projects}

This section lists the five year deliverables (less if the project was defined to be of less than five years) for each of the SIMA projects initiated in FY 1994.

\subsubsection{Manufacturing Systems Environment \\ Control Systems Architecture}

\section{FY94}

Publish a draft report (or several related reports) describing the joint architecture (for manufacturing production systems and equipment). Publish separate reports for architectural specifications (including detailed interface specifications) and methodology.

\section{FY95}

Hold a demonstrable implementation of the joint architecture. Publish a report on the definition of performance metrics for control systems architectures.

\section{FY96}

Publish a report on the initial implementation of the joint architecture. Demonstrate a completed implementation of the joint architecture. Publish a report on the application of performance metrics to the architecture.

\section{FY97}

Publish a report that describes Version 2 of the joint architecture, and evaluates its implementation.

\section{FY98}

Publish a report proposing standards for machine control system architectures.

\section{Integration Specifications}

\section{FY94}

Publish a report summarizing the evaluation of the integration of software applications for mechanical product design and manufacturing planning using object brokering mechanisms. Include a recommendation for implementing this integration approach for design and planning applications.

Publish a document describing the modifications to the Manufacturing Systems Integration (MSI) Control Entity Interface (CEI) specifications for implementation within a industry developed testbed.

\section{FY95}

Create a reference implementation of integrated software applications for mechanical product 
design and manufacturing planning applications using object brokering mechanisms, models of mechanical product information, and models of mechanical process information.

Create an implementation of integrated production systems using the MSI CEI specifications. Evaluate models of information required for this implementation against models specified for use by integrated mechanical product design and manufacturing planning software applications, and publish a requirements document.

\section{FY96}

Demonstrate a production implementation of the CEI specifications at a industry partner facility. Create a specification for algorithms to synthesize commands between the Object Management Group (OMG) Interface Definition Language (IDL) and the $C$ high level programming language for use in integration of software applications using object brokering mechanisms.

Design an integration server which brings together object request brokers serving locally integrated mechanical design and manufacturing software applications. Create a reference implementation of interconnected object request brokers each providing services to a local set of manufacturing applications.

\section{Planning Applications}

\section{FY94}

Hold workshop to refine project plans, define needs, and sign collaborators. Develop functional and information requirements specification for planning application. Publish survey of available manufacturing resource capability models. Extend and refine process plan model specification (ALPS). Install at least one commercial planning system at NIST.

\section{FY95}

Develop preliminary communications protocol specification for planning-design. Develop preliminary standardized capability models of selected manufacturing resources. Define process models for selected manufacturing processes. Hold demonstration of integration of at least one commercial planning system with other commercial engineering and production systems.

\section{FY96}

Publish communications protocol specification for design-planning-production. Refine and extend capability models of manufacturing resources.

\section{FY97}

Develop initial application protocol (AP) definition for shop-level planning (facilitation role).

\section{FY98}

Submit for ISO approval shop-level AP (committee draft). Demonstrate project results. 


\section{Design Applications}

\section{FY94}

Develop document which includes methods to link designs of individual components to enable the application of compute-intensive analysis tools (e.g., assembly sequencing) in verifying and validating assembly requirements.

\section{FY95}

Develop prototype "proof of concept" system to validate assembly representations and direct activities toward development of specifications enabling standards generation.

\section{FY96}

Establish a community of industry, government, academe, and professional organizations that utilize advanced communication utilities to promote design activities leading to an improved product realization cycle.

\section{FY97}

Provide the mechanism for the incorporation of "Design for Produceability" methods and tools into conventional CAD applications.

\section{FY98}

Provide method enabling small-to-medium manufacturers to access design information, knowledge, and services that were previously not obtainable due to a lack of HPCC capabilities.

\section{Process Plant Engineering and Construction}

\section{FY94}

Develop test case of process plant structural system including design and analysis data. Review existing STEP application reference models based on test case and propose needed extensions or refinements.

\section{FY95}

Review the state-of-the-art in technologies applicable to virtual construction. Develop use-case scenarios for a virtual construction environment for the design and construction of process plant structural systems. Establish CRDAs/grants with commercial and academic research centers.

\section{FY96}

Conduct collaborative research with commercial and academic research centers. Complete functional specifications for the interfaces and data repositories in a virtual construction environment.

\section{FY97}

Demonstrate use of virtual construction environment to design for constructability in a process plant context. Develop framework for linking between virtual construction environment and real 
construction environment, e.g., to positioning systems, men, and equipment.

\section{FY 98}

Implement technology transfer to the industry through workshops, standards activity, and CRDAs.

Document the results of the program.

Interoperability of Chemical Measurements and Data Systems

\section{FY94}

Port NIST/EPA/NIH Mass Spectral Database, including search software, to UNIX environment to serve as a network data server to external applications.

Complete development of CAALS-I Communication Specification.

\section{FY95}

Port chemical databases and related property prediction software to a UNIX environment for access through a computer network.

Develop conceptual models for both instrument device capabilities and the steps in analytical chemical methods. STEP and associated tools will be used to specify the form.

\section{FY96}

Develop methods for conducting property-oriented similarity searches to retrieve the most relevant data for specific queries using input both chemical structural and textual information.

Implement a model for device capability datasets (a database specifying the systems available capabilities of a particular instrument) and write the language for building chemical analysis scripts.

\section{FY97}

Devise methods for measuring the reliability and performance of individual components of the developing chemical property retrieval/estimation/extrapolation system.

Combine the scripting language and the capabilities database schema developed thus far with a script/engine/scheduler/sequencer program to create the requirements and specifications for Task Sequencer Controller for controlling an analytical chemistry workcell made up of Standard Laboratory Modules.

\section{FY98}

Test and make available fully functioning chemical property system. It will have the following capabilities: 1) retrieve selected data in NIST chemical property and spectroscopic collections, 2) estimate properties with NIST-developed software, 3) pre-process and route requests to thirdparty data collections and estimation programs, 4) post-process, and when necessary, extrapolate results from other data sources to the conditions of interest. 


\section{Electronic Commerce for the Electronics Industry}

\section{FY94}

Establish a testbed for electronic commerce demonstration development and coordination. Produce an Electronic Business Reply Card demonstration. Sponsor workshops and working meetings as needed (working meeting planned for 2/15-16/94). Give demonstrations and presentations at related conferences (planned participation at Electro 94, CALS Expo and the Design Automation Conference).

\section{Virtual Design Technology}

\section{FY94}

Complete preliminary work underway in modeling measurement system of the Vertical Work Station (VWS) for real-time error correction in precision machining.

Complete a comparative case study on the use of different modeling tools for VWS characterization. Modeling tools compared will include neural networks and statistical regression analysis.

Initiate solicitation of industrial feedback on implementation of modeling technologies for error compensation via machine characterization as part of the specification of an advanced measurement system to realize greater machining precision.

\section{FY95}

Begin preparation of prototype machine characterization software modules, based on research and industrial feedback completed in FY 1994, using Dataplot source code and neural network algorithms.

Begin preparation of machine characterization documentation for electronic dissemination, and identify industrial collaborators for testing and critique of machine characterization software.

\section{FY96}

Complete prototype machine characterization software modules and documentation.

\section{FY97}

Modify machine characterization software and documentation based on industrial user's test results and update or add software modules to support industrial users' up-to-the-minute needs.

\section{FY98}

Complete integration of machine characterization software modules and documentation and make these products available to industrial users through network interface with the NIST manufacturing testbed. 
Packaging and Interconnect Materials Supporting HPCC Infrastructure

(Polymer Process Monitoring for Improved Product Quality)

\section{FY94}

Procure and integrate new LCR meters into current dielectric measurement capabilities. Demonstrate use for measuring dielectric constant and loss from $10^{-3} \mathrm{~Hz}$ to $10^{8} \mathrm{~Hz}$ on substrate resins used for printed wiring boards.

\section{FY95}

Establish means for measuring the dielectric properties of substrate and encapsulant materials over a wide range of frequencies at controlled elevated temperatures and perform measurements on industry-defined materials.

\section{FY96}

Establish and demonstrate means for studying dielectric properties of substrate and encapsulant materials as a function of exposure to environmental agents such as water and processing chemicals.

\section{FY97}

Examine dielectric properties of materials used in substrates and encapsulants as a function of processing conditions and subsequent ageing and exposure to environmental agents.

\section{FY98}

Incorporate dielectric property data as a function of processing conditions, environmental exposure, and ageing into a data base accessible to the microelectronics industry by electronic dissemination.

\section{FY94}

(Effects of Moisture and Temperature on Dimensional Stability)

Introduce through the appropriate standards organizations and in cooperation with the microelectronics industry, an improved standard test method for coefficient of thermal expansion of polymer thin films used in packaging and interconnects as measured by thermal mechanical analysis.

Improve the sensitivity of micrometer-scale changes in dimension of polymeric packaging materials by designing and constructing a cell to measure thermal expansion of thin polymer films based upon changes in capacitance.

\section{FY95}

Establish a testing methodology for measuring the apparent out-of-plane coefficient of thermal expansion of a polymer multilayer assembly employed in the microelectronics industry by developing appropriate test structures and utilizing technical capabilities of thermal mechanical analysis. 
Develop a system for measuring out-of-plane sub-micrometer strain of thin polymer films using a constructed capacitance cell and apply the method to test structures which simulate freestanding and attached dielectric layers used in electronic packaging.

\section{FY96}

In cooperation with the U.S. microelectronics industry, develop data on thermo-mechanical properties of polymeric substrate and encapsulant materials and determine influence of processing history on such properties.

Develop out-of-plane thermal expansion data using a capacitance method on electrically conductive polymer adhesives and on test structures that simulate flip-chip underfill adhesives.

\section{FY97}

Develop in concert with industry and university cooperators, an improved standard test method for introduction through the appropriate standards organizations for measuring the apparent outof-plane coefficient of thermal expansion of attached polymer thin films using the capacitance techniques.

\section{FY98}

Develop out-of-plane thermal expansion data using capacitance and other techniques for test structures that simulate a high performance polymer integrated optical interconnect structure in the absence and under the influence of imposed optical fields.

Develop in conjunction with industry and university partners a standard test method for measuring the out-of-plane coefficient of thermal expansion of integrated optical polymer waveguides and interconnect structures.

\section{FY94}

(Mechanisms of Adhesion of Polymers)

Obtain data on the glass transition temperature and thermal expansion coefficient of several polymer/substrate pairs to explore the effect of adhesion energy on the thermal properties of the overlaid polymer films.

\section{FY95}

Extend neutron reflectivity measurements showing preferential accumulation of moisture at a polymer/silicon interface to other interfaces commonly encountered in electronic package applications such as polymer/chromium and polymer/aluminum.

\section{FY96}

Identify the material parameters which control the amount of moisture accumulation at polymer/substrate interfaces.

Develop a methodology for determining the work of adhesion between a polymer film and a solid substrate based upon measuring the contact area of a micrometer-scale droplet of deformable 
polymer against a substrate under controlled loading.

\section{FY97}

Establish a direct measuring technique for the work of adhesion between polymer films and solid substrates and obtain data on polymer/substrate pairs commonly encountered in electronic packaging.

\section{FY98}

Establish a quantitative relation between the work of adhesion and the material parameters of the constituents at an interface.

\section{FY 94}

(Measurement Techniques for Strain Distribution in Advanced Interconnect Structures)

Assess mechanical and fatigue resistance and generate data for metal conductors commonly used as microelectronic interconnects. Concentrate on techniques necessary to measure quantities used as input in analyses carried out to predict manufacturability and reliability of advanced interconnect structures.

Initiate program development which will allow thermal resistance of unique microelectronic interconnects to be determined both experimentally and by predictive methods based on material properties.

\section{FY95}

Establish precision and bias of thermal conductivity measurement capability and make measurements on unique material forms found in advanced electronic interconnect structures.

Measure local strains produced by thermal stressing and thermal cycling of plated-through-holes and buried vias in copper-solder-board configurations commonly used as microelectronic interconnects.

\section{FY96}

Introduce standard test methods based on newly-developed mechanical and thermal test techniques. This includes identification of industrial collaborators, preparation of drafts for standard test methods, and organizing round robin test series.

\section{FY97}

Begin to make available to industry standards for description and verification of analytical procedures for predicting the manufacturability and reliability of advanced interconnect structures. The predictions would be based on the properties of the materials of construction and the service environment.

\section{FY98}

Begin procurement, distribution, and laboratory testing of a series of interconnect test structures as standard reference materials. This set of test structures should include features relevant to 
various types of interconnect, including optical, electronic digital, electronic analog, and microwave.

\section{Collaborative Environment Platform}

\section{FY94}

Complete the design, procurement and integration of a high performance platform using a ATM LAN and two high performance graphics workstations having interactive audio/video conferencing capability.

\section{FY95}

Demonstration of public domain collaborative environment software. Demonstration of information retrieval for data, images and multi-media using newly developed indexing techniques. Public demonstration at NIST of collaboration for use by companies of all sized.

\section{FY96}

Expand platform for remote operation using ATM WAN and resolve performance problems. Public demonstration of remote collaboration at other sites. Publication of NIST IR and/or journal articles on use of collaborative environments, including solution to commonly encountered problems. Interconnect network with heterogeneous workstations and resolve the interoperability problems.

\section{FY97}

Completion of collaborative environment interoperability study and proposal of any needed standards committee. Provide laboratory support to standards committee for assuring interoperability. Expand platform to provide measurement of performance capabilities as needed. Measure the performance limitations imposed by the concurrency requirements, propose solutions and publish report.

\section{FY98}

Completion of data compression study and proposal for resolution for any needed standards to appropriate standards committees. Publication of final report on collaborative environment study.

\subsubsection{Technology Transfer Environment}

\section{Manufacturing Information Technology Transfer}

\section{FY94}

Define framework for electronically publishing SIMA documents (TTE architecture document). Publish transition plan for technology movement from research through CRADAs with industrial partners (including RMTCs), and MOUs with other government agencies. Hold workshop to refine technology transfer plans. Publish state-of-the-art survey on information dissemination technology. 


\section{FY95}

Publish set of metrics for effectiveness of technology transfer services. Make available SIMA documents via HPCC technology. Hold workshop to define "Collaboratory" design. Design a "Collaboratory" in a specific domain.

\section{FY96}

Publish evaluation of usage of technology transfer services. Establish Collaboratory.

\section{FY97}

Publish evaluation of Collaboratory success. Hold workshop to identify training and education partners. Publish refined plans for information dissemination and collaboratory efforts. Define technology transfer plans for selected SIMA projects.

\section{FY98}

Demonstrate and transfer technology information from other SIMA projects, using tools and techniques developed in project. Establish training and education program.

\subsubsection{Standards Development Environment}

\section{Application Protocol Development Environment}

\section{FY94}

Publish architecture for Application Protocol Information Base (APIB) services and interfaces. Design mechanisms for remote retrieval from APIB.

\section{FY95}

Implement APIB to support remote retrieval of STEP information resource models and initial set of AP`s.

\section{FY96}

Expand APIB to include AP supporting material and work-in-progress on emerging AP's.

\section{FY97}

Demonstrate access to APIB from vendor-supported AP component development tools.

\section{FY98}

Implement mechanisms to support software generation for AP implementation.

\section{Conformance Testing}

\section{FY95}

Develop software that automates test harness functions. Publish certification procedures.

\section{FY96}


Implement product developer's test toolkit in a CASE environment. Publish accreditation procedures.

\section{FY97}

Demonstrate robust test system for transfer to test labs. Perform validation of test suite development tools, Complete test result registration repository.

\section{FY98}

Perform validation of testing harness. Transfer test system to test laboratory. Establish network of test centers, and complete technology transfer to vendors and user community.

\section{STEP for the Process Industry}

\section{FY94}

Refine the research plan for developing process plant STEP application protocols with NISThosted workshops and feedback from industry advisors. Establish U.S. consensus on top level process plant application activity model and document proposed extensions and refinements. Complete Group 1 documentation for STEP AP identified as highest priority by workshop participants and industry advisors.

\section{FY95}

Document "state of IT practices" and information interchange requirements in the process plant industries. Define process plant information interchange requirements to meet ISO 9000 quality standards and OSHA process safety requirements. Complete the working draft for the first process plant STEP AP. Organize U.S. industry reviews of draft process plant STEP APs and other STEP APs relevant to the process plant industries. Initiate second process plant STEP AP.

\section{FY96}

Establish collaborative research project to improve information interchange between detailed design and fabrication of piping systems. Complete Group 1 documentation for the second process plant STEP AP. Continue ISO standardization of process plant STEP APs. Evaluate current IT tools and CAx systems implementations of STEP APs and determine voids and weaknesses which must be resolved to accomplish concurrent engineering and improved productivity in the process plant industries.

\section{FY97}

Complete the working draft of the second process plant STEP AP. Test process plant STEP APs for utility and completeness for re-work of piping systems and re-design of process plants. Test and demonstrate the use of STEP AP(s) for distributed design and fabrication of process plant piping systems. Document test results and recommendations.

\section{FY98}

Test process plant STEP APs for assessing process plant safety and compliance to OSHA requirements. Document test results and recommendations. Test and demonstrate the use of 
process plant STEP APs for process plant certification and maintenance. Document test results and recommendations. Complete technology transfer to the industries through workshops and demonstrations. Document results of the project.

\section{Application Interface Development Environment}

\section{FY94}

Complete the design of the Application Interface Development Environment.

\section{FY95}

Conduct workshop with industry to review requirements for standards and integration and augment identified standards.

Identify interfaces needed to integrate and test standards, and begin development of specific testing tools for selected high priority standards.

Generate report specifying the status of testing tools. These testing tools will be targeted at existing interface standards such as SQT, IRDS, RDA, PHICS, CGM, and STEP.

\section{FY96}

Beta test specific testing tools which were developed, and begin production of an environment for identifying and/or developing the additional standards needed. Demonstrate application of standards across interfaces.

\section{FY97}

Completion of testing tool environment. Utilize standards development environment to identify additional needed standards which will either be (1) referred to the appropriate standards committee or (2) developed in-house.

\section{FY98}

Publish final report on standards development environment.

\section{High Speed Electronic and Optical Components}

\section{FY94}

Develop measurement methods for determining the impedance characteristics of electronic interconnections operating at microwave frequencies.

Complete a round-robin measurement comparison with industry to diagnose the industry's current capability for connector-ferrule inside and outside diameter measurements for optical-fiber connectors.

Determine the appropriate optical-fiber configuration for an NIST Standard Reference Material to support measurements of chromatic dispersion in optical fibers. 


\section{FY95}

Enhance impedance characterization methods to allow characterization of crosstalk in multiple lossy, high-speed, electronic interconnections. Demonstrate utility of methods using commercial components. Submit methods for adoption as industry standards.

Develop metrology for microwave characterization of interconnections using time-domain instrumentation. Demonstrate methods using commercial components. Submit methods for adoption as industry standards.

Develop measurement methods and standards to support connector-ferrule geometry measurements for optical-fiber connectors, and lay the basis for a measurement reference standard.

Complete and analyze an industry-wide round-robin measurement intercomparison to access industry's ability to determine the frequency response of high-speed optical detectors used in lightwave communications systems.

\section{FY96}

Develop measurement methods for characterizing electronic components and multichip modules containing both digital and analog/microwave components.

Develop time-domain calibration and measurement methods useful for accurately characterizing signal fidelity in interconnected digital systems.

Develop measurement methods and standards for characterizing the noise performance of opticalfiber amplifiers used in lightwave communications systems.

\section{FY97}

Demonstrate utility of measurement methods for characterizing electronic components and multichip modules containing both digital and analog/microwave components. Submit methods for adoption as industry standards.

Demonstrate utility of time-domain characterization methods using commercial products. Submit methods for adoption as industry standards.

Develop measurement methods and standards to support high-resolution optical time-domain reflectometry for components used in lightwave communication systems with emphasis on localarea networks.

\section{FY98}

Develop measurement methods for characterizing electronic circuits containing optical as well as digital and analog microwave components.

Develop the measurement techniques and standards to support wavelength-division multiplexed 
lightwave communications components and systems.

\section{Video Processing}

\section{FY94}

Establish a laboratory to permit the testing of displays. Develop and evaluate laboratory tests of various proposed techniques to encode video information so that it is usable in both computing and entertainment environments.

\section{FY95}

Continue development of technology-independent display test and provide automated display evaluation services for industry. Continue evaluation of compatible video encoding methods, and develop objective measures of video quality for rating encoding methods.

\section{FY96}

Complete rapid display-testing procedures, and develop a robotics system for complete panel characterization at "production line" speeds.

\section{FY97-FY98}

Extend display tests to evaluate display performance where it is strongly related to human vision models, for example, artifact visibility, ease of viewing, and other factors.

\subsubsection{AMSANT Facilities}

\section{AMSANT for Mechanical Parts Production}

\section{FY94}

Establish operating testbed in MEL to support MSE projects. Establish $100 \mathrm{Mbps}$ local area network communication speeds for a limited set of internal NIST users supporting SIMA projects. Establish 1 Mbps communication speeds linking NIST to at least three external collaboration partners.

\section{FY95}

Install local network management software for AMSANT. Create software routines within the network management software which proactively identifies network problems. Increase the number of NIST projects supported by $100 \mathrm{Mbps}$ local area network speeds. Increase the number of external collaborations using the $1 \mathrm{Mbps}$ wide area network capability.

\section{FY96}

Install $155 \mathrm{Mbps}$ local area network capability at NIST for a subset of SIMA projects. Broaden coverage of $100 \mathrm{Mbps}$ to cover all remaining SIMA projects. Install $100 \mathrm{Mbps}$ communications speeds directly to a limited set of workstations. Establish $24 \mathrm{Mbps}$ communications link with collaboration partners. Increase the number of collaboration partners to which NIST communicates via $1 \mathrm{Mbps}$ wide area communications speeds. 


\section{Operator Interfaces for AMSANT}

\section{FY94}

Hold conference of industrial manufacturing managers and supervisors to discuss real-world problems and requirements for interfaces and publish proceedings. Hold conference of Virtual Reality (VR) experts to survey state-of-the-art and discuss possible problems and methods for application to manufacturing interfaces and publish proceedings. Develop initial manufacturing operator interface demonstration problems

Publish requirements specification for first generation AMSANT operator interface. Develop specification of initial requirements for VR laboratory. Implement conversions between display database and manufacturing databases. Publish document specifying display database structure and interfaces to AMSANT manufacturing databases for initial manufacturing operator interface.

\section{FY95}

Implement initial manufacturing operator interface and demonstrate working prototypes. Develop and publish specifications for an advanced operator interface system with advanced visualization, displays, and controls. Demonstrate AMSANT real-time visualization laboratory on line, with VR and other advanced visualization and interface technologies. Publish feasibility study of multi-user shared environment technology in application to operator interface visualization database with recommendations.

\section{FY96}

Demonstrate a working advanced manufacturing operator interface system. Develop and publish specifications of extensions to advanced operator interface specifications, such as multi-sensor fusion, force-reflection, tactile-feedback and other modalities. Hold second biennial conference of experts to survey state of the art in visualization technologies and discuss possible problems and methods for application to manufacturing interfaces and publish proceedings.

\section{FY97}

Demonstrate multi-sensor extensions integrated with existing visualization technology. Demonstrate multi-user interactive shared environment database technology.

\section{FY98}

Demonstrate real-time interactive visualization and other sensor modalities for multiple users interacting with the same database objects. Develop and publish report of performance and evaluation measures for advanced manufacturing operator interface. Publish report assessing performance and effectiveness of advanced manufacturing operator interface.

\section{AMSANT for Process Plants}

\section{FY94}

Create the laboratory facility, including at least two commercial software packages that address process plant design/construction. Develop data dictionary for the installed packages. Build 
representations of a process piping system in installed systems.

\section{FY95}

Link the local AMSANT for Process Plant Industries to other AMSANT facilities within NIST. Implement the application reference model, developed in the companion project: STEP for the Process Plant Industries, in an object-oriented database with direct translator-access based on the data dictionary developed in FY 1994.

\section{FY96}

Link the local AMSANT for Process Plant Industries to other AMSANTs outside of NIST. Implement a prototype virtual construction environment in support of the companion project: Process Plant Engineering and Construction. Conduct experiments in support of the companion project: STEP for the Process Plant Industries.

\section{FY97}

Convert the database interfaces to full-performance Standard Data Access Interface (SDAI). Conduct experiments with the virtual construction environment in support of the companion project: Process Plant Engineering and Construction.

\section{FY98}

Demonstrate open-system data access and exchange among participating vendors. Document the software developed and experience gained in the AMSANT.

\subsection{Deliverables for FY95 Incremental Projects}

This section lists the deliverables for each of the new projects within SIMA to be initiated in FY 1995. These deliverables are in addition to the five year deliverables defined in section 6.3 which relate to the projects initiated in FY 1994.

\subsubsection{Manufacturing Systems Environment}

\section{Manufacturing Systems Engineering Tools}

Identify industry needs, the current state-of-the-art, and key technical issues for manufacturing production system design tools. Establish a baseline computing environment using existing commercial off-the-shelf and/or research based engineering tools. Develop a functional requirements document for an integrated computing environment for production system design. Specify a computer-based model of the production system design process for a selected part family.

\section{Advanced Apparel Manufacturing}

Define the set of application protocols needed for apparel manufacturing. Specify the framework for an apparel design system, and develop specifications for the initial implementation of an 
apparel production cell.

\section{Electronic Commerce for the Mechanical Parts Manufacturing}

Define the set of commerce applications relevant to the manufacturing environment. Publish the specifications for the information requirements for the commerce applications.

\section{Interoperability for Chemical Process Measurements and Data Systems}

Complete second level of communication standards between chemical measurement systems. Make available additional database for mass spectrometry of chemicals of industrial interest through the communication networks with appropriate interactive software that will allow manufacturers to easily integrate data into product development. Extend a conceptual model for defining chemical measurement parameters, thus establishing a basis for compatibility for a broad range of chemical measurement instrumentation.

\section{Process Plant Conceptual Design}

Develop a reference model for the comprehensive process plant information base. Test the reference model against existing project definition and conceptual planning methodologies. Use the reference model to define a project historical information base. Demonstrate the use of a prototype implementation to define a process plant project.

\section{Application Protocols for Electronic Products}

A consortium of developers and reviewers will be formed, and work will begin on extending the hybrid Application Protocol (AP) into a layered electrical product AP. By the end of the first year, an activity model should be completed and an information model should be well under way.

\section{Electronic Commerce for the Electronics Industry}

A white paper will be written addressing an open architecture for on-line catalogs, and how to apply this to a national component parts catalog. These ideas will be used to develop electronic commerce projects and level 1 services. Demonstration scenarios will be developed with regional and national network initiatives, such as the Manufacturing Technology Centers and Smart Valley. The EBRC demonstration will be bought forward into a level 1 service.

\section{Integration of Materials Performance Data into Computer-Aided Design}

Select application area for integrating materials information with CAD; to be done in concert with industrial partners. Develop model of materials information requirements for CAD in the selected application area. Plan development of prototype linking existing materials databases and expert systems to CAD system. 


\section{Open-Systems Manufacturing of Enabling Materials}

Develop modeling and systems architecture components suitable for super highway data exchange between designers and manufacturers of structural parts made of polymer matrix composites.

\section{Integration of Reference Data Systems into Information Networks}

Make available selected data from the seven data centers within the Physics Laboratory for access by means of the communications networks. Establish an initial query-based public interface to this data by means of Mosaic client/server access. Enable database searches by industry of some of these databases by means of appropriate computer interfaces.

\subsubsection{Technology Transfer Environment}

\section{Manufacturing Information Technology Transfer for Apparel Production}

Develop specification of requirements for the electronic library, select systems hardware, and identify initial contributors. Develop plan for implementing education outreach program. Establish electronic technology transfer mechanism between NIST AMSANT and the industry supported AMSANT's. Publish strategic plan for increasing and improving government laboratory interactions through the use of HPCC technology. Publish SOA report addressing online knowledge bases and their implications for deploying HPCC into mainstream manufacturing operations. Establish strong collaborations through the development of CRADA's with technology developers, and manufacturing systems users in order to meet the long term objectives of the SIMA program.

\section{Technology Transfer of HPCC information into the Electronics Industry}

Create the initial draft of documentation necessary to teach industry what they need to know about the key electrical/electronic product data standards. All documents will be provided in a network based hypertext format. The initial draft of a series of classes and tutorials will also be outlined. Each course will be targeted at different businesses and at audiences with different level of technical understanding. The project will investigate teaching courses through on-line live video conferencing through ISDN-based software.

\subsubsection{Standards Development Environment}

\section{Harmonization of Standards}

The activities will focus on (1) publishing a plan that identifies the overall standards requirements for harmonization between STEP and EDI, and (2) publishing a plan that identifies the specification for the system that will be required to handle both engineering and commerce type data. 
Create testbed in an open environment based on the Application Portability Profile. Integrate a basic set of software tools in the testbed. Demonstrate operation of the testbed to industry.

\section{Concurrent Engineering of Information Exchange Standards}

Draft Migration Guide and send out for review by industry. Identify communication paths between other initiative teams and the information requirements for those teams. Coordinate the development of documentation archives with all teams. Begin work on the iterative process of developing an engineering $\mathrm{C}++$ class library and concurrent electrical engineering tools.

\section{Infrastructure for Interoperable Electrical/Electronic Product Data Exchange Standards}

Initiate and lead an IEC TC93 working group on testing, and draft a consensus document on testing methodology and requirements. A strategy for meeting the communities test needs will be drafted. A demonstration showing collaborative design and manufacturing through standard interfaces will be drafted.

\subsubsection{AMSANT Facilities}

\section{AMSANT for Apparel Production}

Activities will include the development of the network architecture, and the publication of the first set of interface specifications for planning, scheduling, and control systems. In addition, the specifications for an apparel manufacturing cell will be developed. State-of-the-art communication mechanisms will be provided to engineering and manufacturing researchers to improve data sharing, software sharing and remote communications with other research colleagues. Desktop conferencing systems will be installed in AMSANT and used by industry collaborators to perform remote experiments on manufacturing software systems to test integration concepts.

\section{SUPPORTING PROGRAMS}

This section describes the on-going projects at NIST that relate to the SIMA program. In addition, there is a description of the programs being created by the other government agencies that are involved in the IITA initiative.

\subsection{NIST Projects in Support of SIMA}

In carrying out the SIMA program, NIST will be able to build upon existing projects that are being supported by either the present base programs or by other agencies. The following is a list of the projects grouped by the four SIMA elements and by the NIST laboratory. 


\subsubsection{Manufacturing Systems Environment}

\section{Manufacturing Engineering Laboratory}

Manufacturing Systems Integration Project (sponsored by Navy Mantech) - Design and implement a reference architecture for discrete machined parts which incorporates an integrated production planning and control environment.

Computer-aided Manufacturing Systems Engineering (sponsored by Navy Mantech) - Develop new technologies, methods, tools, standards and collaborative efforts which are required to implement computer-aided tools for engineering manufacturing systems of the future.

Application Protocol for Inspection Planning - Enhance the STEP Part (Shape Tolerance Model) and develop an Application Protocol for the inspection planning process.

PDES for Apparel (sponsored by DLA Mantech) - Develop a comprehensive specification for sharing apparel product data throughout the entire product life cycle.

Persistent Object Bases for Manufacturing (sponsored by ARPA) - Validate the applicability of the new data management technology for future information systems which use the emerging standard STEP.

Enhanced Machine Controller (sponsored by Navy Mantech) - Specify, implement and demonstrate an open architecture machine controller in a machining center testbed.

\section{Chemical Science \& Technology Laboratory}

Architecture for Modular Control and Standard Communications Interfacing of Analytical Instrumentation (sponsored by The Consortium on Automated Analytical Laboratory Systems (CAALS)) - Investigate standardized control strategies and common communication protocols that can provide extensive compatibility between different manufacturer's instrumentation in an automated chemical analysis system.

Structure and Properties Software - Develop special software for the searching and management of chemical databases.

\section{Electronics \& Electrical Engineering Laboratory}

Electronic Commerce of Component Information (ECCI) (joint industry-government effort) Enable the commerce of design, simulation, manufacturing, quality, and test information through a network protocol.

Microwave and Millimeter Wave Advanced Computational Environment (MMACE) (sponsored by DoD Tri-Service Program) - Create industry standards for representing vacuum electronic 
design and manufacturing information.

\section{Materials Science and Engineering Laboratory}

Standards for Technical and Scientific Data (joint with the NIST Office of Measurement Services) - Develop standards for building and accessing databases, and exchanging data among them in the area of chemical and materials data.

\section{Building and Fire Research Laboratory}

Building Industry Framework for Developing STEP Application Protocols - Develop the planning structure, methods, and tools for defining and testing STEP application protocols for the building industry.

Computer-aided System Integration for the Building Industry - Develop methods for application integration that use object technology for interfaces among integrated computer services and combine these methods with the semantic modelling methods developed for STEP.

Computer-based Building Regulations and Standards - Develop a framework for integrating different development and application representations of building regulations and standards in one unified model.

STEP for the Shipbuilding Industry (sponsored by Navy and Navy/Industry Digital Data Exchange Standards Committee) - Test and

validate the conceptual models that establish the shipbuilding requirements for information representation and exchange.

\section{Computing \& Applied Mathematics Laboratory}

Dataplot Software R\&D - Provide an interactive capability for experiment design, data analysis, and modeling to support engineering design.

Process Modeling and Optimization (sponsored by NSF) -

Develop models for specific process design requirements at the cutting edge of manufacturing technology with recent emphasis has been on the processing of ceramic materials, ultra precision grinding and machining of parts, the more precise measurement of surface quality.

\subsubsection{Technology Transfer Enviro nment}

\section{Manufacturing Engineering Laboratory}

Process Planning Testbed (sponsored by ARPA) - Provide information services, workshops, a laboratory for testing and integration, and a collaborative research program for the process planning community. 
STEP On-Line Information Service - Provide access to all the key STEP documents as well as all the STEP related standards.

\section{Materials Science \& Engineering Laboratory}

Systems for Reliable Materials Information (jointly with Office of Measurement Services and Chemical Science \& Technology Laboratory) - Develop a number of databases and expert systems with evaluated information on materials performance.

\section{Physics Laboratory}

Data for Modeling Advanced Manufacturing Processes (jointly with Office of Measurement Services and Chemical Science \& Technology Laboratory) - Provide chemical and physics databases to model advanced manufacturing techniques for producing new materials, either bulk or coatings.

\subsubsection{Standards Development Environment}

\section{Manufacturing Engineering Laboratory}

IGES/PDES/STEP Administration Office - Provide leadership (through the chairmanship) in the national and international standards activities to develop STEP.

National PDES Testbed (sponsored jointly by DoD/CALS and NIST) - Accelerate the development, testing, and implementation of the STEP standards.

Conformance Testing for STEP (sponsored jointly by DoD/CALS and NIST) - Develop and provide a conformance testing service for implementors, government agencies and academia for the chosen application protocols.

\section{Electronics and Electrical Engineering Laboratory}

Electrical/Electronic Product Data Exchange Standards - Facilitate the harmonization of the established standards which can be used to transfer data among automated tools for fabricating electronic products.

\subsubsection{AMSANT Facility}

\section{Manufacturing Engineering Laboratory}

Rapid Response Manufacturing - Establish collaborative research efforts in response to the need for skills and technologies for ensuring the advancement of RRM capabilities throughout U.S. industry. 
Manufacturing Design Research Testbed (sponsored by ARPA/MADE) - Provide an environment for studying the design process, understanding how design information can be represented and determining how to make design information available to necessary systems throughout a product's life cycle.

\subsection{Other Agency Manufacturing Programs}

Both the HPCC Program and the Gore Bill place heavy emphasis on collaboration and the interdependencies between the efforts of individual agencies. This will clearly be a key issue in the manufacturing applications because there are many related efforts underway or planned, particularly within ARPA (Manufacturing Automation Design Engineering, Agile Manufacturing, Persistent Object Bases), other DoD elements (CALS/PDE, FCIM), and DoE (Advanced Manufacturing Technology Network/Sandia Labs and Technologies Enabling Agile Manufacturing/Y12).

It will be part of NIST's strategy to coordinate and build upon these other efforts rather than replace them. To this end, the SIMA program will identify relevant on-going and planned efforts, and, wherever possible, attempt to forge collaborative efforts that leverage the investments and expertise of the various parties involved.

In an effort to work with the other government agencies, standards bodies, industry, and universities, NIST will use the following strategy:

o Perform research and development into PDE and FCIM technologies in collaboration with other institutions;

o Perform research and development into the technologies required to develop the standards;

o Implement the prototype advanced manufacturing systems that conform to the standards;

o Take an active role in developing required standards;

o Take a lead role in developing the testing and evaluation methods to ensure that quality standards are developed and implemented by vendors;

o Work with industrial facilities in implementing the FCIM and PDE standards.

The following is a list of the proposed programs from the various government agencies in manufacturing under the IITA Initiative.

Department of Energy - Manufacturing

The challenges faced by DoE to perform cost effective manufacturing in small lot sizes mirrors 
the challenges facing the U.S. manufacturing community in general. The collection of existing manufacturing related capabilities include networking, computational science, data processing, model-based control, intelligent machines, information surety, telepresence and collaborative work environments that must be deployed to produce a world leading U.S. manufacturing capability.

A revolutionary manufacturing industry will be produced in which designers, manufacturing engineers, marketeers, managers and financial experts and customers of American companies distributed around the world collaborate on the development of products, simulation of manufacturing, optimization of cost or time to market, and automatically set into motion widely dispersed manufacturing, procurement and documentation systems.

A major goal of the program is to establish four national centers for transferring DoE expertise.

\section{National Aeronautics \& Space Agency - Aerospace Design and Manufacturing}

Under this effort NASA will expand its ongoing efforts with industry and academia in the area of multidisciplinary design of aeronautical airframes and aircraft engines to develop an integrated product/process development capability that will significantly reduce product development cycle time while increasing product quality and robustness. A key component of this integrated product/process development capability will be the creation of new capabilities to advance manufacturing technologies in the U.S.

The rationale for this effort is to increase the U.S. economic competitiveness by developing a computer integrated system that will minimize product development cycle and product life cycle cost for a given capability, maximize capability for a given cost, and provide new insight and understanding into advanced manufacturing processes.

The NASA contribution to this program will be to develop, refine, test and transfer advanced computer-integrated electronically networked NASA technologies and applications in the areas of multidisciplinary design and optimization, computational fluid dynamic and structural mechanical simulations to the manufacturing sector.

The goal of this effort is to develop a computer based system to simultaneously design product requirements and specifications, its design optimization, product manufacturing, and product support at the conceptual design level to minimize product life cycle cost for a given capability or to maximize capability for a given cost. The bulk of the life cycle cost is committed during the early phases of the product development process while the bulk of the costs is incurred much later during the manufacturing, operation and support phases of the product. It is therefore imperative to have an Affordable System Optimization Process (ASOP) that can, at the conceptual design level, provide the U.S. industry with products meeting the shortest time cycles between identifying a market opportunity and delivering a product that is required by the market.

A major goal of the program is to establish a working arrangement with an Aerospace consortium of the major companies. 
The program focuses on the development and demonstration of key software elements for Integrated Product/Process Development (IPPD) and agile manufacturing applications within the emerging National Information Enterprise (NIE). The application focus is on mechanical parts and electro-mechanical assemblies, where today's automation environment is neither integrated nor flexible compared to electronics design and manufacturing.

The Manufacturing And Design Engineering (MADE) program will address the following shortcomings:

(1) Gaps in the spectrum of tools for IPPD. Most of today's mechanical CAD/CAE/CAM tools and models apply to the detailed configuration design stage, and focus on product (rather than process) design. MADE will develop tools for conceptual design that provide a spreadsheet-like capability for iterative optimization of product and process characteristics. The initial focus will be on design for assembly and assembly process planning, simulation, and control.

(2) Lack of interoperability among tools. The principal problem is lack of sharable representations of products and processes at multiple levels of abstraction. MADE will develop and demonstrate self-describing, reusable, sharable product and process representations that can be implemented using persistent object bases. MADE will demonstrate unambiguous interchange of geometry, dimensions, and tolerances in machine interpretable form, and will develop productivity-enhancing capabilities for capturing and sharing data requiring human interpretation, such as design intent. This will provide a foundation for enhancement of emerging product data interchange standards such as STEP.

(3) Inadequate infrastructure for distributed design and manufacturing. Although network connectivity and bandwidth are rapidly improving, there remains a need for a layer of integration services to enable widely distributed engineering and manufacturing applications to interoperate. Today, ad hoc bridges must be constructed for each pair of toolsets used within the distributed design team, and for each pair of manufacturing systems within the multi-tiered production team. The results are severe scaling problems as the number of pairs increases. MADE will demonstrate a scalable capability to share multiple types of distributed information among networked applications that were not explicitly designed for interoperability. MADE will prototype a layer of network integration services using intelligent agents to facilitate interactions among coarse grained objects (encapsulated legacy systems), fine grained objects (such as new $\mathrm{MADE}$ tools) and man-in-the-loop applications. Initial services will include brokering of services for engineering analysis and manufacturing processes, limited electronic commerce support, and multi-media interchange of information among engineering and manufacturing applications.

\section{National Science Foundation - Advanced Manufacturing}

The Computer \& Information Science Engineering (CISE) and Engineering are the two lead directorates in support of research in information and computer technologies for advanced 
manufacturing. The main emphasis of this element is to bring a variety of component hardware and software technologies to bear on the next generation of manufacturing. These include databases, networks, communications, and other information infrastructures to be integrated into the entire manufacture cycle of design, production, marketing, and service. Ongoing program activities range from large engineering research centers, interdisciplinary groups, to individual investigators. Strong coupling with industry in joint research, education, and professional training is encouraged.

Major goals for FY 1994 include (1) support new research in the integration of databases, realtime sensing and control, networking, and multi-media and visualization techniques for interactive design and other manufacture processes, (2) initiate cross-disciplinary programs to support work in virtual environments, telerobotics, and telepresence, and other innovative technologies for collaborative design and other manufacturing processes, and (3) develop programs to support new research in salability of technologies applied to manufacturing.

Major goals for FY 1995 include (1) initiate demonstration projects in virtual environments technology and its application in virtual factory based on NREN, (2) develop programs to improve transition of academic research to industry by fully exploring the information infrastructures, including the NREN, and (3) develop programs for training the next generation of manufacturing engineers in the information age.

\section{PROGRAM MANAGEMENT}

The SIMA program is very much unique at NIST. This is a program involving all eight research laboratories who each have a mission to support specific industries and technologies. It takes quite an effort to coordinate the various interests into one consolidated program that addresses the issues of systems integration for manufacturing applications. There is a SIMA council that consists of representatives from each of the laboratories that serves as a consensus body to ensure that the Program is accomplishing its goals. In addition, NIST has a role as the lead agency in the coordination of agency programs that relate to the IITA manufacturing area. This will require the development of MOUs with the various agencies and the ultimate creation of a vision and plan that allows for the leveraging of the activities across the Government. It is also planned to form CRDAs with the key industry consortia who have programs that complement the activities of the Government IITA programs. In order to accomplish this last objective, the SIMA program will form a SIMA Network of participants who will join together to share both the research \& development of manufacturing applications as well as the testing, implementation, and demonstration of HPCC-enriched manufacturing systems over a national communications network.

SIMA will be directed by the Program Manager who is a member of the Factory Automation Systems Division of the Manufacturing Engineering Laboratory. The Program Manager will be responsible for (1) providing technical and administrative leadership to the Program, (2) planning and executing collaborative, multi-year technology development and deployment projects and tasks that are responsive to the legislation and to the needs of U.S. industry, (3) ensuring that the 
Program is a focal point for coordinating efforts in response to the legislation, (4) ensuring that results of the research are implemented in a timely and effective manner, and (5) identifying related on-going and planned technology development and deployment efforts by Federal agencies, States, industries, and Academe.

The Program Manager will be assisted in managing the Program by four Coordinators, one for each of the four elements of the Program. The Coordinators will report to the Program Manager and will provide technical leadership to the activities being carried out in his/her program element. The Program Manager and Coordinators will work closely with other organizational units within NIST which are contributing to the HPCC and will draw upon the staff of other organizational units in order to plan and execute the Program.

The Program Manager will interface with the NIST laboratories participating in the SIMA program using the following coordination structure:

Objective: Provide effective management to the Systems Integration for Manufacturing Applications (SIMA) Program.

Premise: The SIMA program will be very visible in the Clinton/Gore administration. It is critical to have a successful program with exciting and relevant deliverables each year. A key measure of program success is the level of acceptance by industry of the SIMA output.

Laboratory Participation: The SIMA program emphasizes the importance of providing information technology to solving the problems of systems integration for manufacturing. An important part of the program is to identify and utilize generic technology, such as data modeling, in the application to a broad range of manufacturing products. For this reason, the program seeks a commitment to a broad NIST laboratory participation.

Baseline: For FY 1994 and FY 1995, the projects and level of funding for each laboratory have been determined and is reflected in the budget submissions.

Responsibility: MEL has the management responsibility and authority for the program direction and laboratory funding.

\section{Procedures:}

o The Laboratory representatives involved in developing the laboratory projects form the Systems Integration for Manufacturing Applications Council (SIMAC);

o The MEL representative will be the chairman of the SIMAC;

o A project that has been approved for a given fiscal year (e.g. FY 1994 and FY 1995) will submit a project plan by the end of the first quarter that will be approved by the SIMAC in order to receive official recognition as a HPCC project; 
o All projects will undergo a review by the SIMAC during the third quarter;

o The SIMAC will plan a all-project demonstration to be held near the end of the fourth quarter. The demonstration plan will be approved by the end of the second quarter;

o The SIMAC will determine whether projects should be continued beyond the present year based on future plans and present performance. Funding could be redistributed among the participating laboratories if a project demonstrates poor performance;

o The SIMAC chairman will be responsible for publishing a yearly program report. Each SIMAC member is expected to participate in this exercise.

\section{PROGRAM STATUS}

The SIMA program received its first phase of funding, \$9M, for the FY 1994 initiative in January, 1994. All the projects are in the planning phase with finalized project plans approved by the end of February, 1994. Efforts have already begun to establish the facilities and obtain necessary hardware and software to meet the program deliverables. Emphasis is also strong in establishing partnerships with other government agencies with common program goals in advanced manufacturing applications. In addition, there are many CRDAs with industrial consortia being worked through in order to establish joint projects. The FY 1995 budget has been submitted by the President and there is $\$ 16.2 \mathrm{M}$ of increased funding expected.

\section{ACKNOWLEDGEMENTS}

This plan is the result of input from many sources. Much of the material relating to the project descriptions and deliverables was coordinated by Mark Luce, the SIMA program manager. The information on the IITA in Section 2 and the other agency program descriptions in Section 7 was coordinated by Selden Stewart who attended most of the IITA working group meetings and was responsible for maintaining the electronic version of the IITA report to HPCCIT. Obviously the descriptions of the specific NIST projects in Section 5 and the deliverables in Section 6 were prepared by the NIST project managers.

\section{REFERENCES}

[AMT93] "Advanced Manufacturing Technology: The FY 1994 Federal Program in Manufacturing Science, Engineering, and Technology," a report by the Committee on Industry and Technology, Federal Coordinating Council for Science, Engineering, and Technology, Office of Science and Technology Policy, August 1993.

[BLO093] Bloom, H.M., "Strategic Plan for the Factory Automation Systems Division," NISTIR 5148, March 1993.

[CARV91] Carver, G.P., Bloom, H.M., "Concurrent Engineering Through Product Data 
Standards," NISTIR 4573, May 1991.

[FCCS93] "FCCSET Initiatives in the FY 1994 Budget", a report by the Committee on Physical, Mathematical, and Engineering Sciences, Federal Coordinating Council for Science, Engineering, and Technology, Office of Science and Technology Policy to supplement the President's Fiscal Year 1994 Budget, April 8, 1993.

[HPCC93] "High Performance Computing and Communications: Toward a National Information Infrastructure", a report by the Committee on Physical, Mathematical, and Engineering Sciences, Federal Coordinating Council for Science, Engineering, and Technology, Office of Science and Technology Policy to supplement the President's Fiscal Year 1994 Budget, published by the National Coordination Office for HPCC, July 2, 1993.

[IITA94] "Information Infrastructure Technology and Applications", A report to the HPCCIT, published by the IITA Task Group, January 4, 1994.

[ISAT93] Working slides from the ARPA ISAT Study on NII Services, held in August 1993.

[NII93] "The National Information Infrastructure: Agenda for Action", published by the Information Infrastructure Task Force, September 15, 1993.

[OTA92] U.S. Congress, Office of Technology Assessment, Global Standards: Building Blocks for the Future, TCT-512 (Washington, DC: U.S. Government Printing Office, March 1992.).

[SINM93] "Needs and Opportunities: U.S. Manufacturers Define the Role of NIST," a workshop sponsored by NIST, August 16-17, 1993, Gaithersburg, MD. 


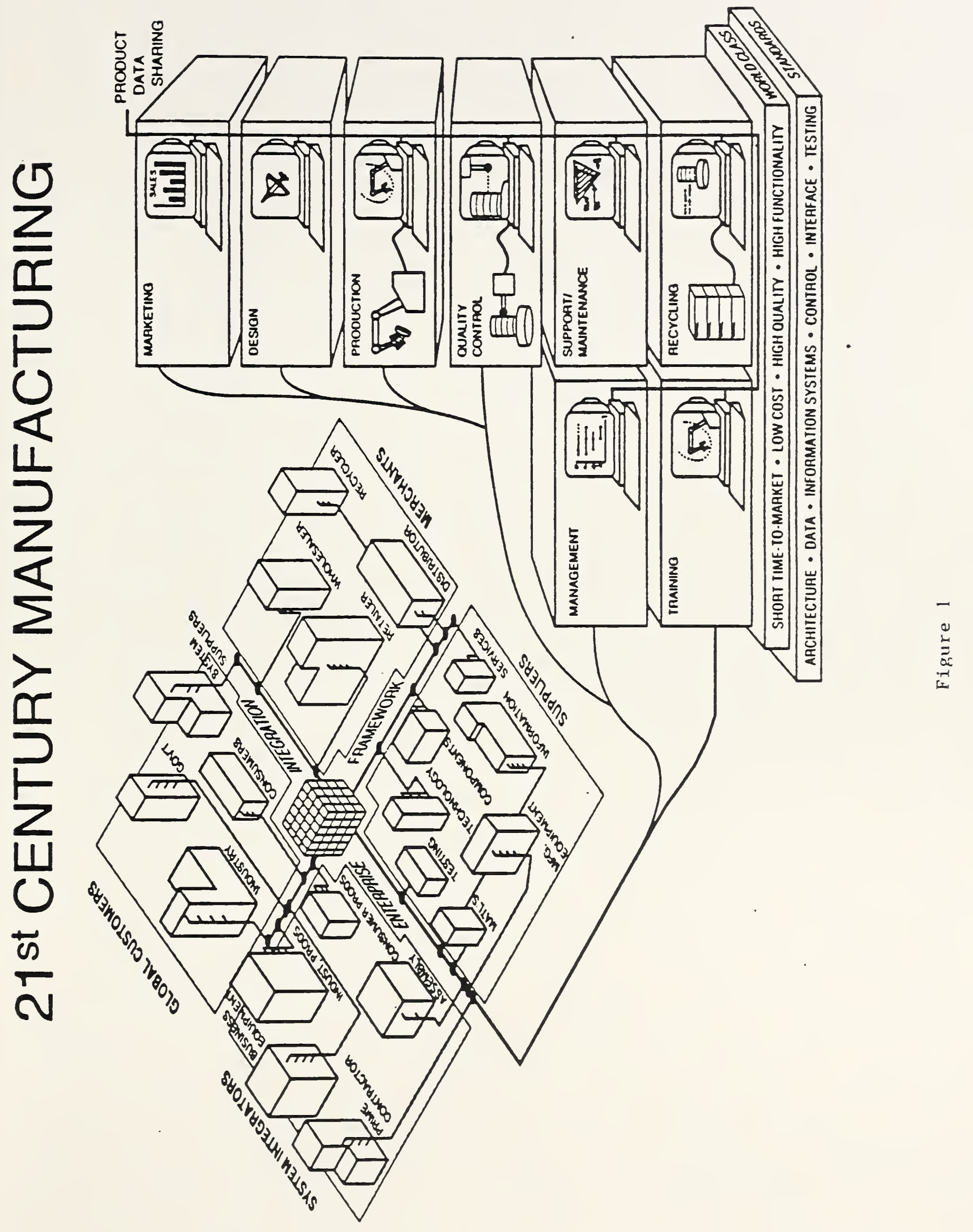



